\section{ENERGY-MOMENTUM RESTRICTIONS ON THE CREATION OF GOTT TIME MACHINES}

\author{
Sean M. Carroll, ${ }^{*}$, Edward Farhi,, Alan H. Guth,,$\S$ and Ken D. Olum ${ }^{\dagger}$
}

Center for Theoretical Physics

Laboratory for Nuclear Science

and Department of Physics

Massachusetts Institute of Technology

Cambridge, Massachusetts 02139 U.S.A.

Submitted to Physical Review D

\section{CTP\#2252, gr-qc/9404065}

April 1994

This paper replaces CTP\#2117, "Gott Time Machines Cannot Exist in an Open (2+1)-Dimensional Universe with Timelike Total Momentum," by S. M. Carroll, E. Farhi, and A. H. Guth. This version is precise about the meaning of "open universe," and proves that if the total momentum of such a universe is timelike then the total deficit angle is no more than $2 \pi$.

* This work was supported in part by funds provided by the U.S. National Aeronautics and Space Administration (NASA) under contracts NAGW-931 and NGT50850, and the U.S. National Science Foundation under grant PHY/9206867.

$\S$ This work was supported in part by funds provided by the U.S. Department of Energy (D.O.E.) under contract \#DE-AC02-76ER03069.

$\dagger$ This work was supported in part by funds provided by the Texas National Research Laboratory Commission under grant \#RGFY93-278C.

\section{ENERGY-MOMENTUM RESTRICTIONS ON THE CREATION OF GOTT TIME MACHINES}

\author{
Sean M. Carroll, Edward Farhi, Alan H. Guth, and Ken D. Olum
}

\section{ABSTRACT}

The discovery by Gott of a remarkably simple spacetime with closed timelike curves (CTC's) provides a tool for investigating how the creation of time machines is prevented in classical general relativity. The Gott spacetime contains two infinitely long, parallel cosmic strings, which can equivalently be viewed as point masses in (2+1)-dimensional gravity. We examine the possibility of building such a time machine in an open universe. Specifically, we consider initial data specified on an edgeless, noncompact, spacelike hypersurface, for which the total momentum is timelike (i.e., not the momentum of a Gott spacetime). In contrast to the case of a closed universe (in which Gott pairs, although not CTC's, can be produced from the decay of stationary particles), we find that there is never enough energy for a Gott-like time machine to evolve from the specified data; it is impossible to accelerate two particles to sufficiently high velocity. Thus, the no-CTC theorems of Tipler and Hawking are enforced in an open (2+1)-dimensional universe by a mechanism different from that which operates in a closed universe. In proving our result, we develop a simple method to understand the inequalities that restrict the result of combining momenta in (2+1)-dimensional gravity.

\section{INTRODUCTION}

Absent some restriction on boundary conditions and energy sources, it is possible for the spacetime metric of general relativity to wreak havoc with our intuitive notions of "going forward in time." We can imagine metrics in which the worldline of a test particle, locally restricted to the interior of its forward light cone, can loop around to intersect itself - a closed timelike curve (CTC). Indeed, it is easy to construct solutions to Einstein's equations that exhibit such behavior [1-3].

Nonetheless, due to the ca usal paradoxes associated with such a time machine, it is tempting to believe that XTC's exist only in spacetimes that are in some way pathological. That is, we wou d expect that the laws of physics somehow act to prevent the occurrence of CTCS in the real universe. This expectation has been dubbed the "Chronology Protection Conjecture" [4] 
In the context of classical general relativity, a counterexample to the chronology protection conjecture would be a solution to Einstein's equations that describes the creation of CTC's, using only ordinary materials, in a local region of a spacetime that is free of CTC's in the past. There is evidence, in the form of theorems proven by Tipler [5] and Hawking [4], that no such solutions exist. These results demonstrate that CTC creation in a local region free of singularities (i.e., with a compactly generated Cauchy horizon) is incompatible with the requirement that only normal matter be used (i.e., that the weak energy condition be satisfied). The Tipler-Hawking theorems, however, leave open the possibility of CTC formation if a singularity appears (rendering the local region of spacetime non-compact). It is tempting to assume that any such CTC's will be hidden behind an event horizon, but that has not been proven. ${ }^{1}$ We review the Tipler-Hawking theorems in Appendix A.

Scientific interest in CTC's has been invigorated by Gott's $[8,9]$ construction of an extraordinarily simple solution to Einstein's equations that contains CTC's. Gott's solution describes two infinitely long parallel cosmic strings moving past each other at high velocity. The situation at early times is portrayed in Fig. 1, which shows the two strings approaching each other. Each string is associated with a deficit angle removed from the space, which we have oriented in a direction opposite to that of the motion of the string. Opposite sides of the excluded wedges are identified at equal times. Gott found that, as the strings approach each other it becomes possible to traverse a closed timelike curve encircling the strings in the sense opposite to their motion. The spacetime is topologically equivalent to Minkowski space ${ }^{2}$ and free of singularities and event horizons.

It is interesting to ask how the Gott solution is reconciled with the TiplerHawking theorems. Although cosmic strings have never been observed, there is no reason to believe that they are unrealistic forms of energy and momentum; certainly they satisfy the weak energy condition, as required for the no-CTC theorems However, the strings in Gott's solution are infinitely long, so the CTC's clearly do not arise in a local region. Thus, in their original form, the theorems of Tipler and

1 Indeed, the cosmic censorship conjecture (on which this assumption is based) has recently been brought into question by numerical simulations [6]. Moreover, Ori [7] has recently argued that the singularities required by the Tipler-Hawking theorems need not prevent the creation of CTC's.

2 In discussing the topology of the Gott universe, we are treating the strings as objects with a small but nonzero thickness. They are nonsingular configurations, and are not excised from the spacetime.

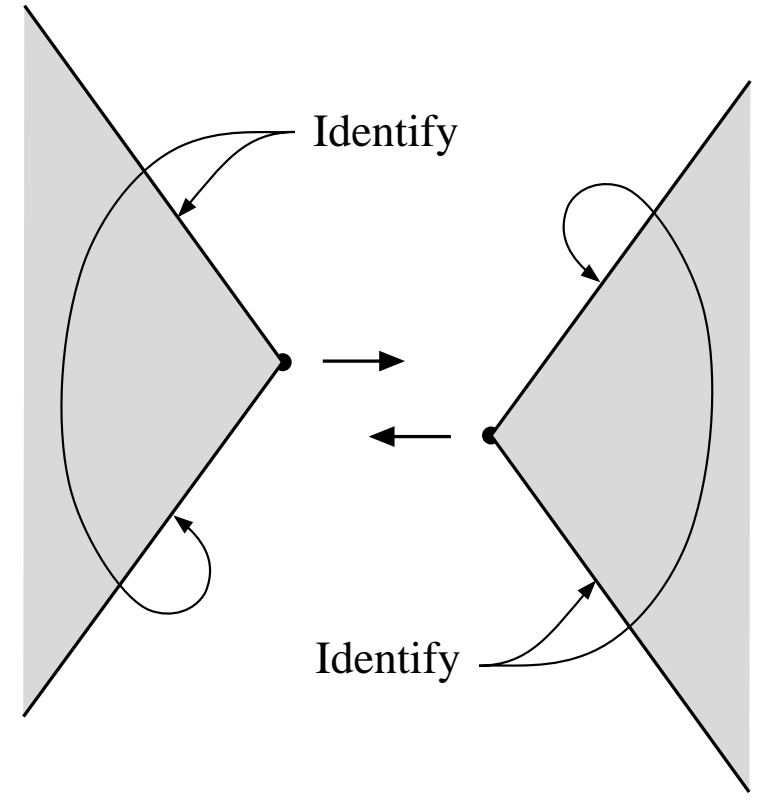

Fig. 1: A spacelike slice through the Gott spacetime. Two parallel cosmic strings perpendicular to the page, represented by dots, move past each other at high velocity. A deficit angle (shaded) is removed from the space around each string, with opposite sides identified at equal times. (If the deficit angles were oriented in any direction other than along the motion of the string, the identifications would be at unequal times.) Note that no CTC's pass through this spacelike surface, as explained in the text.

Hawking have nothing to say about the Gott time machine.

With further analysis, however, it can be seen that the infinite length of the strings does not free the Gott spacetime from the implications of arguments similar to those of Tipler and Hawking. The relevance of the theorems can be established by exploiting a special feature of the Gott spacetime: its equivalence to a spacetime with point masses in $(2+1)$ dimensions. Any $(3+1)$-dimensional spacetime populated solely by infinitely long parallel cosmic strings is invariant under boosts and translations along the direction of the strings, so the trivial dependence of the metric on this direction can be ignored. The strings are then described as particles in $(2+1)$ dimensions, and the Gott time machine consists of two particles moving toward each other at high speed. Although the Tipler-Hawking theorems were originally proven in the context of $(3+1)$-dimensional general relativity, they may be extended to the $(2+1)$-dimensional case, as we discuss in Appendix A. The 
reconciliation of these theorems with the Gott spacetime, therefore, involves issues more subtle than the infinite length of the strings.

In an investigation of the causal structure of the Gott spacetime, Cutler [10] showed that it contains regions free of CTC's, and constructed a complete spacelike hypersurface for which there are no CTC's in the past. A simpler example of such a hypersurface is shown in Fig. 1; the past light cone of any point on this equal-time surface extends through similar surfaces arbitrarily far into the past, implying that no timelike curve through such a point can be closed. As the two particles approach one another, each will ultimately collide with the trailing deficit angle of the other, at which time this coordinate system will fail; CTC's will then arise. These CTC's, however, do not originate in a compact region of spacetime; the chronology horizon (the boundary of the region containing CTC's) extends indefinitely in the past direction, although it never intersects the hypersurface of Fig. 1 . Thus even when we consider Gott's spacetime as a (2+1)-dimensional universe of point particles, the CTC's are still not compactly generated.

Nevertheless, in order to explore how classical general relativity complies with the Tipler-Hawking theorems, one can imagine attempting to construct a Gott-like time machine. Suppose, in a (2+1)-dimensional universe previously free of CTC's, that two particles are accelerated toward each other in an attempt to reach the velocity needed for a Gott time machine. Since the consequences of this acceleration would be confined to the future of the region in which it occurs, the chronology horizon could not extend indefinitely toward the past, as it does for the full Gott spacetime. In analogous situations in $(3+1)$ dimensions, it is conceivable that the creation of CTC's is permitted, and the theorems merely imply that singularities are produced in the process. If so, the CTC's may or may not be hidden by event horizons. Alternatively, the creation of CTC's might be strictly forbidden. One could imagine, for example, that any attempt to create a time machine would be thwarted by energy loss due to gravitational radiation. In $(2+1)$ dimensions, however, neither singularities nor gravitational radiation can occur. The theorems of Tipler and Hawking imply, however, that some mechanism must prevent the formation of a Gott time machine in these circumstances. Because these (2+1)dimensional systems are exactly solvable, we will be able to study this mechanism in detail.

The $(2+1)$-dimensional theory has been the object of extensive investigation $[11,12]$. It has been found that the metric in vacuum is necessarily flat, ${ }^{3}$ while in the presence of a single particle with mass $M$, the external metric is that of Minkowski

\footnotetext{
3 We will always assume that the cosmological constant vanishes.
}

space from which a wedge of angle $\alpha=8 \pi G M$ has been removed and opposite sides have been identified ( $G$ is Newton's constant). Solutions with several static particles are easily constructed by joining several one-particle solutions, in which case the space has a net deficit angle given by the sum of the deficit angles of the constituent particles. If the total deficit angle of a space with static particles exceeds $2 \pi$, then the spatial sections of the spacetime must be closed [13]; the topology is $S^{2}$, and the total deficit angle is necessarily exactly $4 \pi$. By joining appropriately boosted single-particle spacetimes, the exact solutions with moving particles [12] and nontrivial decays and scatterings [14] may be constructed.

In their seminal paper on (2+1)-dimensional gravity, Deser, Jackiw, and 't Hooft [12] noted that a spinning point particle would give rise to CTC's. They added, however, that "such closed timelike contours are not possible in a space with $n$ moving spinless particles, where angular momentum is purely orbital." When stated without qualification, this sentence is apparently contradicted by the existence of the Gott solution. What is true, as implied by the $(2+1)$-dimensional version of the Tipler-Hawking theorems, is that CTC's cannot be created from scratch in a local region. The mechanism by which these theorems are enforced will be the main subject of this paper.

The energy and momentum of a collection of particles can be conveniently characterized by the Lorentz transformation that a vector undergoes upon being parallel transported around the system $[12,15]$. (In the presence of CTC's we must be careful in choosing the loop which surrounds the particles, as we explain below.) The Lorentz transformation belongs to the $(2+1)$-dimensional Lorentz group, $\mathrm{SO}(2,1)$. Deser, Jackiw, and 't Hooft [16] have shown that the group element corresponding to the Gott time machine is boostlike, i.e., equivalent under similarity transformation to a pure boost. Each element of $\mathrm{SO}(2,1)$ can be identified with a 3-vector, which we will refer to as the energy-momentum vector of the system. The energy-momentum vector for a single particle is timelike, while that of the Gott two-particle system is spacelike (tachyonic), despite the fact that each particle is moving slower than $c$.

The possibility of timelike momenta combining to form a spacelike momentum arises because the momentum of a system of particles is not the sum of the individual momenta. In (2+1)-dimensional gravity, unlike special relativity, the composition law for energy-momentum vectors is nonlinear. Hence it becomes possible for a system composed of ordinary, subluminal matter to have the same energy-momentum vector as a tachyonic particle. ${ }^{4}$

\footnotetext{
4 The energy-momentum vector, however, does not tell the whole story. The
} 
One might guess that this discovery reveals why it is impossible to build a Gott time machine from slowly-moving particles: because the momentum is tachyonic, and we can exclude tachyons as unphysical. In fact, we will argue that the momentum of Gott's time machine is not unphysical: it is possible, given sufficient energy, to produce a Gott pair from the evolution of initially static particles, even in a theory that does not contain fundamental tachyons.

There are, nevertheless, insuperable barriers to creating a Gott time machine, by which we mean a system of particles with spacelike total momentum that leads to the creation of CTC's. In an open universe, the obstacle is that there is never sufficient energy. In our previous paper [14] we examined a specific scenario the decay of two initially static particles in an open universe - and showed that the offspring particles could never move fast enough to make a Gott time machine. In this paper we generalize the result, by examining the evolution determined by arbitrary initial data specified on an edgeless spacelike surface $S$. A necessary condition for a Gott time machine to evolve from such data is that a subsystem of particles in the domain of dependence of $S$ (or on the boundary of the domain of dependence) have a spacelike momentum. We show that if $S$ has the topology of $\mathbb{R}^{2}$ and the total momentum passing through $S$ is timelike, then there is insufficient energy for a spacelike subsystem to arise. In this sense, a Gott time machine cannot be created in an open universe with timelike total momentum.

As the key step in our demonstration, we associate with every collection of particles an element of the universal covering group of $\mathrm{SO}(2,1)$, and show that an element corresponding to an open hypersurface with timelike momentum is never the product of an element representing the Gott time machine and any number of elements representing massive particles. The proof can be constructed by algebraic manipulation; however, an elegant geometric demonstration is achieved by introducing an invariant metric on the parameter space of the group, in which case the group manifold becomes $(2+1)$-dimensional anti-de Sitter space. ${ }^{5}$

In a closed universe, this result no longer holds - we have found that it is possible to construct a spacetime in which a pair of particles with tachyonic momentum is created from initially static conditions (i.e., by the decay of massive particles). However, 't Hooft [17] has shown that causal disaster is avoided in this

external spacetime associated with a fundamental tachyon is different from that associated with a Gott time machine, as we will make explicit in Sec. II.

5 We are very grateful to Don Page and Alex Lyons, who pointed out to us the relationship between the $(2+1)$-dimensional Lorentz group and anti-de Sitter space. case, since the universe shrinks to zero volume before any CTC's can arise. ${ }^{6} \mathrm{He}$ goes on to argue that this phenomenon will always result from an attempt to build a time machine in a closed universe. Therefore, neither closed nor open universes in $(2+1)$ dimensions can evolve Gott time machines from initial conditions with slowlymoving particles. These cases provide concrete illustrations of the mechanisms that enforce the theorems of Tipler and Hawking.

\section{ENERGY AND MOMENTUM}

\section{A. Overview}

This section is devoted to a detailed discussion of the use of holonomy the Lorentz transformation associated with parallel transport of a spacetime vector around a closed loop - to quantify the energy and momentum of gravitating point particles in $(2+1)$ dimensions. With a suitable choice of coordinate system, the holonomy is a simple function of the velocities and deficit angles of the particles enclosed by the loop.

The parameter space of $\mathrm{SO}(2,1)$, the group of Lorentz transformations in $(2+1)$ dimensions, can be given an invariant metric, establishing a correspondence between the group manifold and $(2+1)$-dimensional anti-de Sitter space. We find that there are inequalities that relate the momentum of a system of particles to the momenta of the constituents, and that these inequalities can be expressed compactly by referring to the causal structure of the anti-de Sitter metric. In the process, we find it useful to extend $\mathrm{SO}(2,1)$ to its universal covering group, in which rotations are not identified modulo $2 \pi$, and which can therefore distinguish between (for example) a universe with no matter and a universe with total deficit angle $2 \pi$ or $4 \pi$.

We wish to comment that many of the tools we use are standard results in the theory of Lie groups and symmetric spaces [18]. Any Lie algebra has a natural metric, the Cartan-Killing form, given in components by

$$
g_{\mu \nu}=c_{\mu \sigma}^{\lambda} c_{\nu \lambda}^{\sigma}
$$

where the $c^{\alpha}{ }_{\beta \gamma}$ are the structure constants. For semisimple groups, this metric can be uniquely extended to a left- and right-invariant metric on the entire group

6 In a note added to our previous paper [14] we erroneously claimed that CTC's would arise. We thank G. 't Hooft for informing us of our mistake. 
manifold. The resulting space will be maximally symmetric, and paths from the identity defined by $T(\lambda)=\exp (-i \lambda X)$, where $X$ is an element of the Lie algebra, will be geodesics of this metric. However, it is straightforward for us to derive these results for the case at hand, which we will do for clarity. (A similar discussion of the universal cover of $\mathrm{SO}(2,1)$ in a different context can be found in Ref. [19]. A more mathematical discussion can be found in Ref. [20].)

\section{B. Holonomy}

We begin by recalling the characterization of energy and momentum in $(2+1)$ dimensional gravity $[12,15]$. In any number of dimensions, spacetime curvature can be characterized by the holonomy transformation that describes the result of parallel transporting a vector around a closed loop. In $(2+1)$ dimensions this technique is especially convenient, since the holonomy of a (contractible) closed loop can be thought of as reflecting the energy and momentum of the matter passing through the loop. Further, since space is flat outside sources, any two loops that can be deformed into each other without crossing any particles will yield the same transformation. (The holonomy will, of course, depend on a choice of frame at the base point of the loop. If the choice of frame is varied, the holonomy will change by a similarity transformation.)

In a general manifold, the holonomy around a loop is a path-ordered exponential of the connection. In the case at hand, however, the flatness of spacetime in vacuum affords a considerable simplification. As an example, consider a single particle that is stationary in a Minkowskian coordinate system from which a wedge of deficit angle $\alpha$ has been removed, with opposite sides identified. A vector parallel transported in a counterclockwise loop around the particle has constant components until it crosses the identified edges, where it undergoes a rotation by $\alpha$. The holonomy of this loop is therefore a counterclockwise rotation matrix $R(\alpha)$. For a particle in a similar coordinate system but moving with velocity $\vec{v}$, the appropriate transformation can be obtained by boosting to the particle's rest frame, rotating, and boosting back. Thus, we associate with the moving particle a matrix

$$
T=B(\vec{\xi}) R(\alpha) B^{-1}(\vec{\xi}),
$$

where $\vec{\xi}=\hat{v} \tanh ^{-1}|\vec{v}|$ is the rapidity of the particle and $B(\vec{\xi})$ is a boost bringing the rest vector to the velocity of the particle.

If there are several particles moving with respect to one another, then the holonomy around any loop can be computed by deforming the loop so that it goes around the particles one at a time. The holonomy is then a product of matrices, each of which has the form of Eq. (2).
The discussion of multiparticle systems is particularly simple in the context of a spacetime (or region of spacetime) free of CTC's, since in this case we can construct a foliation into spacelike surfaces. Specifically, the momentum of a system of particles contained in a bounded, simply connected region of a spacelike surface is characterized by the holonomy of the (counterclockwise) loop that forms the boundary of the region. (We consider the particle worldlines to be part of the manifold, so such worldlines do not render a region multiply connected.) The base point of the holonomy can be thought of as the position of the observer, and defines the coordinates in which the holonomy transformation is measured. The relation between this holonomy and the properties of the individual particles is easily seen by continuously deforming the loop into one that encircles the particles one at a time, as shown in Fig. 2. Such a deformation can always be constructed by first choosing non-intersecting paths $P_{i}$ to connect each particle to the base point $Q$. (In a coordinate system adapted to this picture, the deficit angles would not cross the paths $P_{i}$ but otherwise may extend in any direction.) The original loop $C$ is then deformed to the loop $C^{\prime}$ by continuously shrinking the area inside the loop, continuing until all parts of the loop come into contact with either the particles or the paths $P_{i}$. The parallel transport of a vector around the path $C^{\prime}$ is thus the product of the parallel transport transformations of the loops around the individual particles, so

$$
T_{\text {tot }}=T_{N} T_{N-1} \ldots T_{1},
$$

where the particles are enclosed in the order $1,2, \ldots N$. Here each $T_{i}$ has the form of Eq. (2), where the velocity of the $i$ th particle is determined by parallel transporting the velocity vector to the base point $Q$ along the path $P_{i}$.

As a system of $N$ particles evolves via decay or scattering into a system of $M$ particles, a loop around the system at one time can be deformed to a loop around the system at a later time (i.e., on a subsequent spacelike surface). Since the deformation carries the loop only through regions of flat spacetime, the resulting transformation matrix is not changed. Therefore, conservation of energy and momentum is expressed as the equality of Lorentz transformations at different times:

$$
T_{N} T_{N-1} \ldots T_{1}=T_{M}^{\prime} T_{M-1}^{\prime} \ldots T_{1}^{\prime} .
$$

This will hold regardless of the orientations of the paths $P_{i}$, as long as the base points of the two loops lie in the same Minkowskian coordinate patch. This rule was used in Ref. [14] in constructing the spacetime for one particle decaying into two.

Later in this paper we will be concerned with the relation between a system of particles and a subset of the system. The subsystem is defined by specifying the 
(a)

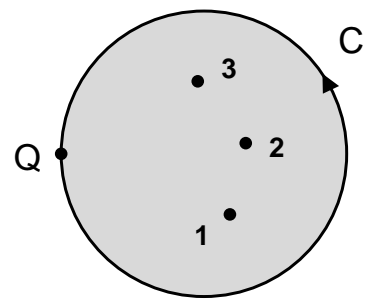

(b)

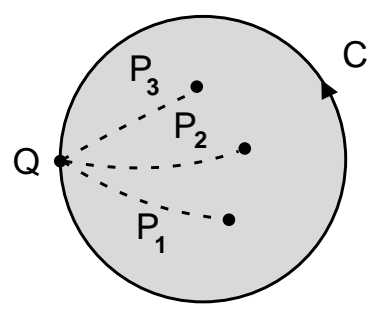

(c)

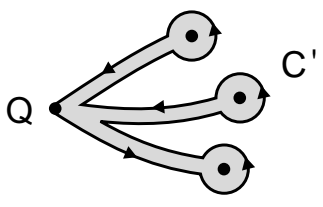

Fig. 2: The deformation of a loop into a succession of single particle loops. Part (a) shows three particles contained in a shaded region, bounded by the counterclockwise loop $C$ with base point $Q$. To deform the loop, first draw non-intersecting paths $P_{i}$ connecting each particle to the base point, as shown by the dashed lines in (b). (These paths are arbitrary, but in a connected region they can always be constructed.) Then deform the loop so that the area inside shrinks, continuing until all parts of the loop come into contact with either the particles or the paths $P_{i}$. The result is a path $C^{\prime}$, as shown in (c), which encircles the particles one at a time.

region of the spacelike surface that it occupies. To obtain a holonomy that can be compared with the holonomy of the full system, the base point $Q$ of the full holonomy should also lie on the boundary of the subregion, and should be taken as the base point for its holonomy. Note that the specification of the region contains more information than a simple listing of the particles in the subsystem; Fig. 3 shows an example of two distinct regions that contain exactly the same particles. To understand the relevance of the extra information, recall that the holonomy (a)

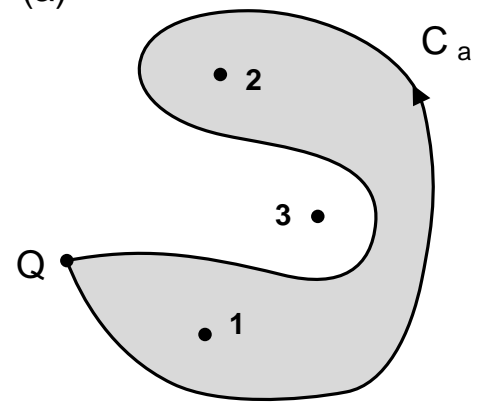

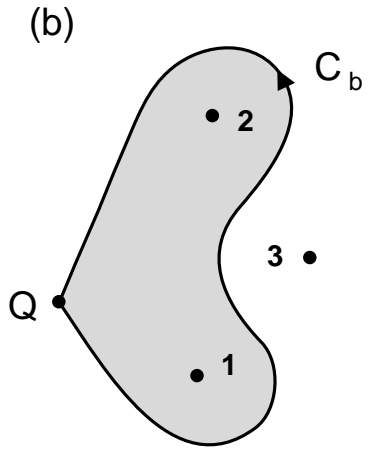

Fig. 3: Two distinct regions containing the same particles. The shaded regions in parts (a) and (b) each contain the same particles, 1 and 2 . Nonetheless, the boundary loops $C_{a}$ and $C_{b}$ cannot be continuously deformed into one another without crossing particle 3 , and therefore the holonomies of the two loops will be unequal.

defines the energy and momentum of the subsystem, and therefore must include a specification of the relative velocity of the two particles. The velocity of particle 2 as seen by particle 1 , however, depends on the path used for the observations. That is, the velocities can be compared only by parallel transporting one to the other, a path-dependent process.

The generic form of a system and subsystem are shown in Fig. 4a. The loop around the system is called $C_{t o t}$, and the loop around the subsystem is called $C_{s u b}$. To relate the total holonomy $T_{t o t}$ to the holonomy of the subsystem $T_{s u b}$, first deform $C_{t o t}$ to $C_{t o t}^{\prime}$, as shown in Fig. $4 \mathrm{~b}$. The deformation consists of adding a detour which runs along $C_{s u b}$, and then returns along the same path. Then note that the loop $C_{\text {tot }}^{\prime}$ can be viewed as a sequence of two loops, as shown in Fig. 4c. The first is a loop $C_{s u b}$, surrounding the subsystem, and the second is a loop $C_{\text {else }}$ that surrounds the remainder of the system. The holonomy for the full system can then be written as

$$
T_{\text {tot }}=T_{\text {else }} T_{\text {sub }} \text {. }
$$

$C_{\text {else, }}$, however, is a loop surrounding a simply connected region, so it can be decomposed into its single particle contributions as in Eq. (3). Thus, $T_{\text {tot }}$ can always be written as

$$
T_{t o t}=T_{N} T_{N-1} \ldots T_{1} T_{\text {sub }}
$$

where the $T_{i}$ denote matrices of the form of Eq. (2), for each particle not part 
(a)

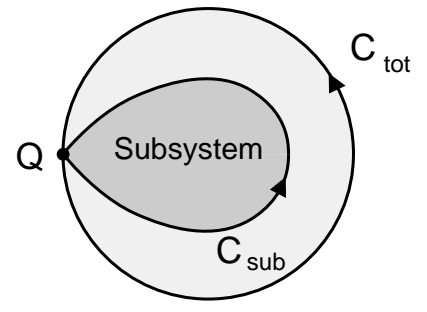

(b)

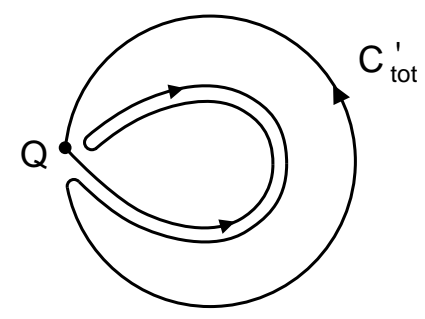

(c)

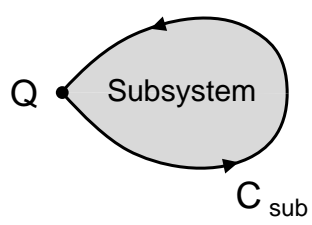

Followed

By

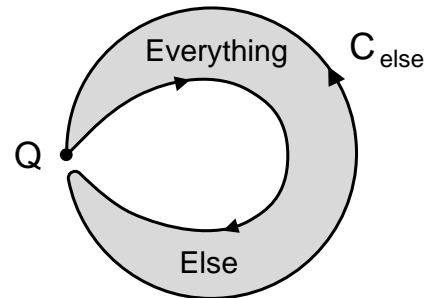

Fig. 4: The relation between a subsystem and the entire system. Part (a) shows a generic system and subsystem, bounded by loops $C_{t o t}$ and $C_{s u b}$, respectively. The loop $C_{t o t}$ can be deformed, as shown in (b), by extending it along the path of $C_{s u b}$ and then back again. Part (c) shows how the new loop, $C_{t o t}^{\prime}$, can be viewed as the concatenation of two loops: $C_{s u b}$, which surrounds the subsystem, and $C_{\text {else, }}$, which surrounds the remainder of the system.

of the subsystem. This equation implies that no matter how the subsystem is chosen, the complete system can always be separated cleanly into the subsystem plus other particles. Eq. (6) will be used later to prove an inequality relating the energy-momentum of a subsystem to that of the system in which it is contained.

We turn now to a specific representation of the holonomy transformations $T$ by $2 \times 2$ matrices. The standard basis for the Lie algebra of $\mathrm{SO}(2,1)$ consists of the rotation generator $J$ and two boost generators $K_{1}$ and $K_{2}$, with the commutation relations

$$
\begin{aligned}
& {\left[K_{1}, K_{2}\right]=-i J} \\
& {\left[J, K_{1}\right]=i K_{2}} \\
& {\left[J, K_{2}\right]=-i K_{1} .}
\end{aligned}
$$

Following the conventions used in Ref. [14], we take $J=\frac{1}{2} \sigma_{3}$ and $K_{i}=\frac{i}{2} \sigma_{i}$, where the $\sigma$ 's are the standard Pauli matrices. When exponentiated, these $2 \times 2$ matrices generate the group $\mathrm{SU}(1,1)$, which is a double cover of $\mathrm{SO}(2,1)$. Since our ultimate concern will be the universal cover common to $\mathrm{SO}(2,1)$ and $\mathrm{SU}(1,1)$ for convenience we will work with $\mathrm{SU}(1,1)$ in what follows.

The generators $J$ and $K_{i}$ are components of an antisymmetric Lorentz tensor $M_{\mu \nu}$, with $J=M_{12}$ and $K_{i}=M_{i 0}$. Since we are working in three spacetime dimensions, however, we can define a set of 3-vector group generators by using the Levi-Civita tensor:

$$
\mathcal{J}_{\mu}=\frac{1}{2} \epsilon_{\mu}{ }^{\lambda \sigma} M_{\lambda \sigma}
$$

where $\epsilon_{012} \equiv 1$, and indices are raised and lowered by the Lorentz metric $\eta_{\mu \nu} \equiv$ $\operatorname{diag}[-1,1,1]$. Explicitly, $\mathcal{J}_{0}=J, \mathcal{J}_{1}=-K_{2}$, and $\mathcal{J}_{2}=K_{1}$.

A rotation by angle $\alpha$ is given by

$$
R(\alpha)=e^{-i \alpha J}=\left(\begin{array}{cc}
e^{-i \alpha / 2} & 0 \\
0 & e^{i \alpha / 2}
\end{array}\right)
$$

and a boost is given by

$$
B(\vec{\xi})=e^{-i \vec{\xi} \cdot \vec{K}}=\left(\begin{array}{cc}
\cosh \frac{\xi}{2} & e^{-i \phi} \sinh \frac{\xi}{2} \\
e^{i \phi} \sinh \frac{\xi}{2} & \cosh \frac{\xi}{2}
\end{array}\right),
$$

where $\xi=|\vec{\xi}|$ is the magnitude of the rapidity and $\phi$ is its polar angle. The matrix $T$ associated with a single particle is found by evaluating Eq. (2):

$$
T=\left(\begin{array}{cc}
\sqrt{1+p^{2}} e^{-i \alpha^{\prime} / 2} & i p e^{-i \phi} \\
-i p e^{i \phi} & \sqrt{1+p^{2}} e^{i \alpha^{\prime} / 2}
\end{array}\right)
$$

where

$$
p \equiv \sinh \xi \sin \frac{\alpha}{2}
$$

is a measure of the momentum of the particle, and $\alpha^{\prime}$ is defined by

$$
\tan \frac{\alpha^{\prime}}{2}=\cosh \xi \tan \frac{\alpha}{2} \text {. }
$$

Note that $\alpha^{\prime}$ may be thought of as a boosted deficit angle, when the excised wedge is taken to lie along the direction of motion (as in Fig. 1). 


\section{The Metric on SU(1,1)}

We now turn to the geometry of the parameter space of $\mathrm{SU}(1,1)$. The space of $2 \times 2$ matrices is spanned by the group generators $\mathcal{J}_{\mu}(\mu=0,1,2)$ and the identity matrix, so an arbitrary $2 \times 2$ matrix can be written as

$$
T=w-2 i \chi^{\mu} \mathcal{J}_{\mu}=\left(\begin{array}{ll}
w-i t & y+i x \\
y-i x & w+i t
\end{array}\right)
$$

where $\chi^{\mu} \equiv(t, x, y)$, and $w, t, x$, and $y$ are complex. $\mathrm{SU}(1,1)$ consists of those matrices $T$ satisfying

$$
\operatorname{det} T=+1
$$

and

$$
T^{\dagger} \eta T=\eta
$$

where

$$
\eta=\left(\begin{array}{cc}
1 & 0 \\
0 & -1
\end{array}\right)
$$

It is shown in Appendix B that these conditions are obeyed if and only if $(t, x, y, w)$ are real numbers satisfying

$$
-t^{2}+x^{2}+y^{2}-w^{2}=-1
$$

The form of this equation suggests that we consider the three-dimensional space indicated by Eq. (15) to be embedded in a four-dimensional space with metric

$$
d s^{2}=-d t^{2}+d x^{2}+d y^{2}-d w^{2} .
$$

It is natural to take the metric on the parameter space of $\operatorname{SU}(1,1)$ to be the metric induced by this embedding. Aficionados of de Sitter spaces will recognize this as $(2+1)$-dimensional anti-de Sitter space (see Ref. [3]). The group $\mathrm{SO}(2,2)$ which leaves this metric invariant will map the submanifold defined by Eq. (17) into itself, so the embedded three-manifold is maximally symmetric. Furthermore, it will be shown in Appendix B that the metric is group invariant - that is, either left- or right-multiplication by an element of $\mathrm{SU}(1,1)$ is an isometry of the metric.

To put coordinates on $\mathrm{SU}(1,1)$, note that we can decompose any element into a boost times a rotation:

$$
\begin{aligned}
T & =B(\vec{\zeta}) R(\theta) \\
& =\left(\begin{array}{cc}
e^{-i \theta / 2} \cosh \frac{\zeta}{2} & e^{i(\theta / 2-\delta)} \sinh \frac{\zeta}{2} \\
e^{i(-\theta / 2+\delta)} \sinh \frac{\zeta}{2} & e^{i \theta / 2} \cosh \frac{\zeta}{2}
\end{array}\right),
\end{aligned}
$$

where $\zeta$ is the magnitude of the boost, $\delta$ is its polar angle, and $\theta$ is the angle of rotation. (Note that this is a different parameterization than the variables $(\alpha, \xi$, $\phi)$ used in Eqs. (11-13).) Comparing (19) to Eq. (14) and defining $\psi=2 \delta-\theta$, we obtain

$$
\begin{aligned}
& t=\sin \frac{\theta}{2} \cosh \frac{\zeta}{2} \\
& x=\sin \frac{\psi}{2} \sinh \frac{\zeta}{2} \\
& y=\cos \frac{\psi}{2} \sinh \frac{\zeta}{2} \\
& w=\cos \frac{\theta}{2} \cosh \frac{\zeta}{2} .
\end{aligned}
$$

The anti-de Sitter metric on $\mathrm{SU}(1,1)$ is the metric induced by Eq. (18) on the submanifold defined by Eq. (17). In the coordinates $(\theta, \zeta, \psi)$, it is obtained by plugging the transformations (20) into Eq. (18), yielding

$$
d s^{2}=-\frac{1}{4} \cosh ^{2} \frac{\zeta}{2} d \theta^{2}+\frac{1}{4} d \zeta^{2}+\frac{1}{4} \sinh ^{2} \frac{\zeta}{2} d \psi^{2} .
$$

Thus $\theta$ acts as a timelike coordinate.

\section{The Energy-Momentum Vector}

Elements of $\mathrm{SU}(1,1)$ sufficiently close to the identity may be written as exponentials of elements of the Lie algebra:

$$
T=\exp \left(-i \phi^{\mu} \mathcal{J}_{\mu}\right)
$$

Since $\phi^{\mu}$ describes a tangent vector at the identity of $\operatorname{SU}(1,1)$, we can compute its norm using the metric defined above. One could use Eq. (21), but it is easier to expand $T$ to first order in $\phi^{\mu}$, and then to compare with Eq. (14) to determine the parameters $(d t, d x, d y, d w)$ needed in Eq. (18). The result is

$$
|\phi|^{2}=\frac{1}{4} \eta_{\mu \nu} \phi^{\mu} \phi^{\nu}
$$

Not surprisingly, the group invariant metric in the vicinity of the identity coincides, up to a factor, with the usual $(2+1)$-dimensional Minkowski metric $\eta_{\mu \nu}$. If $|\phi|^{2}<0$ we will call the vector "timelike," keeping in mind the distinction between the timelike direction in the spacetime manifold and that on $\operatorname{SU}(1,1)$. Note that $|\phi|$ is 
actually the length of the curve defined by $T(\lambda)=\exp \left(-i \lambda \phi^{\mu} \mathcal{J}_{\mu}\right)$, where $\lambda$ varies from 0 to 1 , as can be seen by first calculating the length of the segment from $\lambda$ to $\lambda+d \lambda$.

To understand the properties of $\phi^{\mu}$, let us write the holonomy of a loop around a single particle in the form $T=\exp \left(-i \phi^{\mu} \mathcal{J}_{\mu}\right)$. Under a Lorentz transformation $L$ in the physical spacetime, the group element $\exp \left(-i \phi^{\mu} \mathcal{J}_{\mu}\right)$ transforms as

$$
L \exp \left(-i \phi^{\mu} \mathcal{J}_{\mu}\right) L^{-1}=\exp \left(-i \Lambda^{\mu}{ }_{\nu} \phi^{\nu} \mathcal{J}_{\mu}\right),
$$

where $L$ is an element of the $2 \times 2$ representation of $\mathrm{SU}(1,1)$ and $\Lambda$ is the corresponding matrix in the $3 \times 3$ (adjoint) representation. In the rest frame of a single particle $T$ is a pure rotation and $\phi^{\mu}$ is equal to $(\alpha, 0,0)=(8 \pi G M, 0,0)$. Using Eq. (24), one sees that in an arbitrary frame $\phi^{\mu}=8 \pi G(\gamma M, \gamma M \vec{v})$, in agreement (up to a factor) with the energy-momentum vector of special relativity. It follows immediately that any massive particle (moving slower than the speed of light) will be associated with a $\phi^{\mu}$ that is timelike. (By convention, the holonomy of a counterclockwise loop corresponds to a future-directed energy-momentum vector.) If several particles are combined, however, then $\phi_{t o t}^{\mu}$ is not the sum of the individual momenta; rather, from Eq. (3)

$$
\exp \left(-i \phi_{\text {tot }}^{\mu} \mathcal{J}_{\mu}\right)=\exp \left(-i \phi_{N}^{\mu} \mathcal{J}_{\mu}\right) \exp \left(-i \phi_{N-1}^{\mu} \mathcal{J}_{\mu}\right) \ldots \exp \left(-i \phi_{1}^{\mu} \mathcal{J}_{\mu}\right)
$$

In the $G \rightarrow 0$ limit, on the other hand, each exponential can be expanded to lowest order, and one finds that $\phi_{t o t}^{\mu}$ approaches the sum of the individual $\phi_{i}^{\mu}$. Since $\phi_{t o t}^{\mu}$ is a conserved Lorentz 3-vector which approaches the ordinary special relativistic energy-momentum vector in the $G \rightarrow 0$ limit, we will call it the energy-momentum vector. However, it should be recognized that it is a somewhat unconventional energy-momentum vector in at least two respects. First, and more importantly for our purposes, the energy-momentum vector for a group of particles is not the sum of the individual momenta. Second, it is constructed from the Lie algebra of the Lorentz group, rather than the tangent space of the spacetime. Nonetheless, in $2+1$ dimensions the Lorentz generators can be rearranged to form a vector, as in Eq. (8). The expansion of a group element in terms of these generators gives rise to a three-tuple $\phi^{\mu}$, which transforms according to Eq. (24) as a vector in the tangent space of the spacetime. The tangent space is constructed at the location of the base point of the closed loop used for parallel transport, which might be thought of as the location of the observer. ${ }^{7}$

\footnotetext{
7 If multiple observers are stretched along the loop, or along any deformation
}

For some purposes it will be convenient to write $\phi^{\mu}$ as a parameter $\lambda$ times a normalized vector $n^{\mu}$; if $\phi^{\mu}$ is timelike $|n|^{2}=-1$, and for $\phi^{\mu}$ spacelike $|n|^{2}=+1$. For $n^{\mu}$ timelike, the explicit form for $T$ is

$$
\begin{aligned}
e^{-i \lambda n^{\mu} \mathcal{J}_{\mu}} & =\cos \frac{\lambda}{2}-2 i n^{\mu} \mathcal{J}_{\mu} \sin \frac{\lambda}{2} \\
& =\left(\begin{array}{cc}
\cos \frac{\lambda}{2}-i n^{0} \sin \frac{\lambda}{2} & \left(n^{2}+i n^{1}\right) \sin \frac{\lambda}{2} \\
\left(n^{2}-i n^{1}\right) \sin \frac{\lambda}{2} & \cos \frac{\lambda}{2}+i n^{0} \sin \frac{\lambda}{2}
\end{array}\right),
\end{aligned}
$$

and for $n^{\mu}$ spacelike we obtain

$$
\begin{aligned}
e^{-i \lambda n^{\mu} \mathcal{J}_{\mu}} & =\cosh \frac{\lambda}{2}-2 i n^{\mu} \mathcal{J}_{\mu} \sinh \frac{\lambda}{2} \\
& =\left(\begin{array}{cc}
\cosh \frac{\lambda}{2}-i n^{0} \sinh \frac{\lambda}{2} & \left(n^{2}+i n^{1}\right) \sinh \frac{\lambda}{2} \\
\left(n^{2}-i n^{1}\right) \sinh \frac{\lambda}{2} & \cosh \frac{\lambda}{2}+i n^{0} \sinh \frac{\lambda}{2}
\end{array}\right) .
\end{aligned}
$$

Taking the trace of these two equations, we can see that a general matrix obtained by exponentiation satisfies

$$
\frac{1}{2} \operatorname{Tr}\left[e^{-i \lambda n^{\mu} \mathcal{J}_{\mu}}\right]= \begin{cases}\cos \frac{\lambda}{2} & \text { for } n^{\mu} \text { timelike } \\ \cosh \frac{\lambda}{2} & \text { for } n^{\mu} \text { spacelike } .\end{cases}
$$

It follows that

$$
\frac{1}{2} \operatorname{Tr}\left[e^{-i \lambda n^{\mu} \mathcal{J}_{\mu}}\right] \geq-1
$$

for all cases.

From Eq. (29), it is easy to show that the SU(1,1) matrix corresponding to the Gott time machine is not the exponential of any generator. For simplicity, we take a configuration where the two particles approaching each other (as in Fig. 1) each have rest frame deficit angle $\alpha$ and rapidity $\xi$, with $\phi_{1}=\pi, \phi_{2}=0$. Then we can use Eqs. (3) and (11) to write $T_{G}=T_{2} T_{1}$ as

$$
T_{G}=\left(\begin{array}{cc}
\left(1+p^{2}\right) e^{-i \alpha^{\prime}}-p^{2} & -2 p \sqrt{1+p^{2}} \sin \frac{\alpha^{\prime}}{2} \\
-2 p \sqrt{1+p^{2}} \sin \frac{\alpha^{\prime}}{2} & \left(1+p^{2}\right) e^{i \alpha^{\prime}}-p^{2}
\end{array}\right)
$$

of the loop that intersects no other particles, then the observers will agree on the energy-momentum vector in the following sense: if the vector measured by one observer is parallel transported along the loop to another observer, it will agree with the vector measured by the second observer. 
where $p$ and $\alpha^{\prime}$ are given by Eqs. (12) and (13). The trace is then given by

$$
\begin{aligned}
\frac{1}{2} \operatorname{Tr} T_{G} & =\left(1+p^{2}\right) \cos \alpha^{\prime}-p^{2} \\
& =1-2 \cosh ^{2} \xi \sin ^{2} \frac{\alpha}{2} .
\end{aligned}
$$

The condition that such a configuration contain closed timelike curves is [8]

$$
\cosh \xi \sin \frac{\alpha}{2}>1
$$

or $\frac{1}{2} \operatorname{Tr} T_{G}<-1$. Thus, from Eq. (29) it follows that $T_{G}$ cannot be written as an exponential.

However, $\mathrm{SU}(1,1)$ is a double cover of the Lorentz group, so the matrices $\pm T_{G}$ correspond to the same element of $\mathrm{SO}(2,1)$. We will see in the next section that $-T_{G}$ can be written as an exponential. Since $\frac{1}{2} \operatorname{Tr}\left(-T_{G}\right)>1$, Eq. (28) implies that it is the exponential of a spacelike generator. The corresponding element of $\mathrm{SO}(2,1)$ can be obtained by exponentiating the corresponding generator, and thus the element of $\mathrm{SO}(2,1)$ associated with a Gott pair is spacelike or tachyonic (equivalent under similarity transformation to a pure boost). This is the sense in which we say that the Gott time machine has tachyonic momentum $[16,14]$, even though $T_{G} \in \mathrm{SU}(1,1)$ is not equivalent to the exponential of a spacelike generator - parallel transport of a spinor around a single tachyonic particle is not equivalent to parallel transport around the Gott two-particle system, although parallel transport of an $\mathrm{SO}(2,1)$ vector does not distinguish between the two cases.

\section{E. Constraints on the Energy-Momentum of Systems}

We now return to the anti-de Sitter geometry of SU $(1,1)$. For fixed $n^{\mu}$, we may consider Eqs. (26) and (27) as defining curves parameterized by $\lambda$. The crucial observation is that these curves are geodesics in the metric (21), which can be checked directly. For example, by comparing Eqs. (19) and (26), one sees that the curve defined by (26) is equivalent to

$$
\begin{aligned}
& \theta=2 \tan ^{-1}\left(n^{0} \tan \frac{\lambda}{2}\right) \\
& \zeta=2 \sinh ^{-1}\left[\sqrt{\left(n^{0}\right)^{2}-1} \sin \frac{\lambda}{2}\right] \\
& \psi=-2 \tan ^{-1}\left(\frac{n^{1}}{n^{2}}\right)=\text { constant } .
\end{aligned}
$$

It is straightforward to confirm that this solves the geodesic equation

$$
\frac{d^{2} x^{\mu}}{d \lambda^{2}}+\Gamma_{\rho \sigma}^{\mu} \frac{d x^{\rho}}{d \lambda} \frac{d x^{\sigma}}{d \lambda}=0
$$

for $x^{\mu}=(\theta, \zeta, \psi)$. This fact can be seen more directly by starting with a simple path, such as $T(\lambda)=\exp \left(-i \lambda \mathcal{J}_{0}\right)$, and verifying that this solves the geodesic equation. Then by Eq. (24) a Lorentz transformation $\Lambda^{\mu}{ }_{\nu}$ will take this curve into another curve of the form $\exp \left(-i \lambda \phi^{\mu} \mathcal{J}_{\mu}\right)$, with $\phi^{\mu}=\Lambda^{\mu}{ }_{0}$. Since the action of $\mathrm{SU}(1,1)$ is an isometry, the resulting curve must also be a geodesic. Finally, the isometry property also ensures that a curve of the form $T(\lambda)=T_{0} \exp \left(-i \lambda n^{\mu} \mathcal{J}_{\mu}\right)$ $\left(T_{0} \in \mathrm{SU}(1,1)\right)$ will be a geodesic through $T_{0}$.

A simple way to visualize anti-de Sitter space is in terms of its Penrose (conformal) diagram [3]. We define a new coordinate $\zeta^{\prime}$ by

$$
\zeta^{\prime}=4 \tan ^{-1}\left(e^{\zeta / 2}\right)-\pi
$$

restricted to the range $0 \leq \zeta^{\prime}<\pi$. The metric (21) becomes

$$
d s^{2}=\frac{1}{4 \cos ^{2} \frac{\zeta^{\prime}}{2}}\left(-d \theta^{2}+d{\zeta^{\prime}}^{2}+\sin ^{2} \frac{\zeta^{\prime}}{2} d \psi^{2}\right)
$$

The Penrose diagram is shown in Fig. 5; the angular coordinate $\psi$ is suppressed. The light cones at each point are lines drawn at $45^{\circ}$. The right hand side of the rectangle is the surface $\zeta^{\prime}=\pi$, which represents spacelike and null infinity. The lower left corner is the origin, from which we have drawn typical spacelike and timelike geodesics. The lower and upper boundaries are the surfaces $\theta=0$ and $\theta=4 \pi$, which are identified (the topology of $\mathrm{SU}(1,1)$ is thus $S^{1} \times \mathbb{R}^{2}$ ). An important feature of this diagram is that timelike geodesics from the origin refocus at the point $\left(\theta=2 \pi, \zeta^{\prime}=0\right)$, as can be seen directly from Eq. (33) (note that $\zeta^{\prime}=0$ is equivalent to $\left.\zeta=0\right)$. Therefore, points that are spacelike separated from $\left(\theta=2 \pi, \zeta^{\prime}=0\right)$ cannot be joined to the origin by a geodesic. These points correspond to the shaded region of the diagram.

Since every element of SU $(1,1)$ that can be written as the exponential of a generator lies along a geodesic through the origin, points in the shaded region correspond to group elements that cannot be reached by exponentiation. The element $T_{G}$ corresponding to the Gott time machine lies in this region. The element $-T_{G}$, on the other hand, can be obtained from $T_{G}$ by subtracting $2 \pi$ from $\theta$, so 


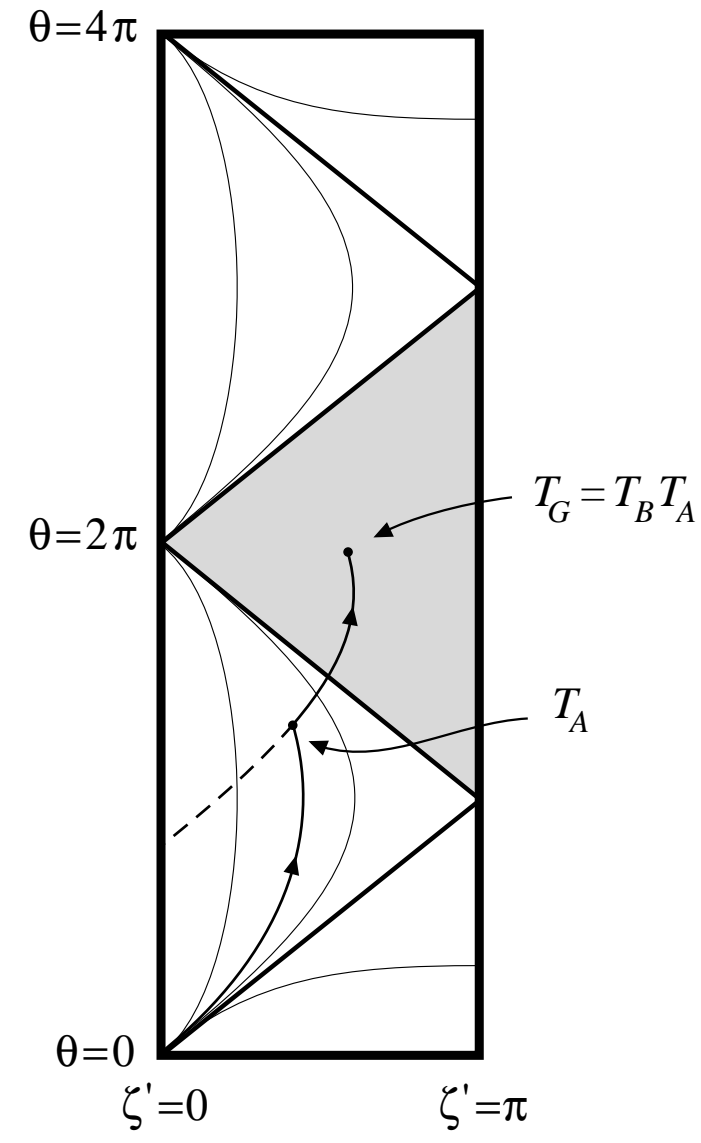

Fig. 5: The conformal diagram of $S U(1,1)$, the double cover of $S O(2,1)$. The group manifold of $\mathrm{SU}(1,1)$ with the invariant metric is shown. The $\psi$ direction is suppressed; hence, each point away from the $\zeta^{\prime}=0$ line represents a circle. The top and bottom edges, $\theta=4 \pi$ and $\theta=0$, are identified. The identity element is in the lower left hand corner; we have indicated some spacelike and timelike geodesics from this point. Elements of $\mathrm{SU}(1,1)$ that can be expressed as exponentials of generators lie on such geodesics. The product $T_{G}$ of two such elements $T_{B}$ and $T_{A}$ is represented by a curve constructed from two consecutive geodesic segments, as shown. In this case the product lies in the shaded region, which represents elements that cannot be expressed as exponentials of generators. The Gott time machine lies in this region.
$-T_{G}$ lies in the region that is spacelike separated from the origin. Thus $-T_{G}$ can be reached by exponentiation, as was claimed in the previous section.

The product of two elements $T_{B}=\exp \left(-i \phi_{B}^{\mu} \mathcal{J}_{\mu}\right)$ and $T_{A}=\exp \left(-i \phi_{A}^{\mu} \mathcal{J}_{\mu}\right)$ corresponds to traveling in the direction of $\phi_{A}^{\mu}$ along a geodesic to $T_{A}$, then traveling along a different geodesic (not through the origin) to $T_{B} T_{A}$. As shown in the diagram, we can easily reach the shaded region, and hence the Gott time machine, in this manner. The parameter space of $\mathrm{SO}(2,1)$ can be visualized by cutting the diagram in half, identifying the surfaces $\theta=0$ and $\theta=2 \pi$. Then the shaded region of Fig. 5 is mapped onto the wedge which is covered by spacelike geodesics emanating from the origin. This is consistent with our earlier statement that the Gott time machine holonomy $T_{G}$, written as an element of $\mathrm{SO}(2,1)$, can be expressed as the exponential of a spacelike generator, even though the $\mathrm{SU}(1,1)$ element for the Gott time machine cannot be expressed as the exponential of any generator.

Consider a system of particles represented by an element $T_{t o t}$ of $\mathrm{SU}(1,1)$. As in Eq. (6), we divide this system into a subsystem represented by an element $T_{s u b}$ and the remaining $N$ individual particles represented by $T_{i}$ :

$$
T_{t o t}=T_{N} \ldots T_{1} T_{\text {sub }} .
$$

This relation can be represented on the Penrose diagram by a future-directed nonspacelike curve from $T_{\text {sub }}$ to $T_{\text {tot }}$, constructed from geodesic segments representing each of the $T_{i}$. It is clear that the periodicity in the $\theta$ direction allows any two points to be connected in this way - as far as $\mathrm{SU}(1,1)$ is concerned, any system of particles can contain a subset with arbitrary energy and momentum. ${ }^{8}$

However, the identification $\theta \leftrightarrow \theta+4 \pi k$ obscures an important difference between physically distinct situations. To make this difference apparent, we must go to $\widetilde{S U}(1,1)$, the universal cover of $\mathrm{SU}(1,1)$ and $\mathrm{SO}(2,1)$. In terms of the Penrose diagram, we no longer identify $\theta=0$ with $\theta=4 \pi$, but instead we extend the picture infinitely far in the positive and negative $\theta$ direction (Fig. 6). The timelike geodesics from the origin will refocus at $\theta=2 \pi$, then continue onward, refocusing again at $\theta=2 \pi k$ for every integer $k$. Therefore the wedges of points that are spacelike separated from $\left(\theta=2 \pi k, \zeta^{\prime}=0\right)$ for $k \neq 0$ cannot be reached from any geodesic

8 Note also that an arbitrary loop, encircling some particles clockwise and others counterclockwise, will correspond to a sequence of both future- and past-directed geodesic segments. However, we will limit our attention to loops that encircle all particles counterclockwise. 
through the origin. All of these points may be said to correspond to tachyonic momenta, since they map to elements of $\mathrm{SO}(2,1)$ lying on spacelike geodesics from the origin.

To describe a multiparticle system using $\widetilde{\mathrm{SU}}(1,1)$, we express $T_{\text {tot }}$ as a product as in Eq. (3), with each $T_{i}$ representing a single particle. Any particle in its rest frame has a deficit angle $\alpha<2 \pi$, so one can uniquely define $T_{i}$ in the universal covering group by using Eq. (2), interpreting $R(\alpha)$ as the element of the universal covering group described by $\left(\theta=\alpha, \zeta^{\prime}=0\right)$, where $0 \leq \alpha<2 \pi$. Similarly $B(\vec{\xi})$ can be chosen to lie in the sector of the universal covering group that is spacelike separated from the identity. (In this case, however, any other choice would be equivalent. The ambiguity consists of any number of factors of the group element corresponding to a rotation by $2 \pi$, and this element commutes with all other elements. Therefore, in Eq. (2), the ambiguous factor would cancel between $B$ and $B^{-1}$.)

We will continue to use the word "holonomy" to refer to the group element $T_{t o t} \in \widetilde{\mathrm{SU}}(1,1)$, although there is no type of physical particle that we could parallel transport around a closed loop which would distinguish between elements of $\widetilde{\mathrm{SU}}(1,1)$ separated by a $4 \pi$ rotation. Unlike a true holonomy, however, the $\widetilde{\mathrm{SU}}(1,1)$ element is not uniquely defined by the loop $C$ alone: it is also necessary to specify, up to continuous deformation, a disk $D_{C}$ of which $C$ is the boundary. This disk allows a unique specification of which particles are inside the loop, so that Eq. (3) can be written. (If the loop $C$ were drawn on an $S^{2}$ surface, for example, there would be two inequivalent areas that it would bound. The two resulting elements of $\widetilde{S U}(1,1)$ would differ by a $4 \pi$ rotation, so the specification of $D_{C}$ is needed to resolve the ambiguity.)

The definition of $T_{t o t} \in \widetilde{\mathrm{SU}}(1,1)$ given above is conveniently explicit, but we must show that the result is independent of the arbitrary paths $P_{i}$ that were introduced to write $T_{t o t}$ as a product of single-particle group elements $T_{i}$, as was done in Eq. (3). For this purpose, we introduce an alternative definition. Recall that an element of the covering group $\tilde{G}$ of any group $G$ is uniquely defined by

Fig. 6: The universal cover of $S U(1,1)$. In the universal covering group, the identification of $\theta$ with $\theta+4 \pi$ is removed, so that $\theta$ takes values from $-\infty$ to $\infty$. The element $T_{G}$, representing a Gott time machine, does not lie to the past of any point on the $\zeta^{\prime}=0$ line between $\theta=0$ and $\theta=2 \pi$. Since all open hypersurfaces with timelike total momentum lie on this line (in their rest frame), no such hypersurface can contain a Gott time machine.

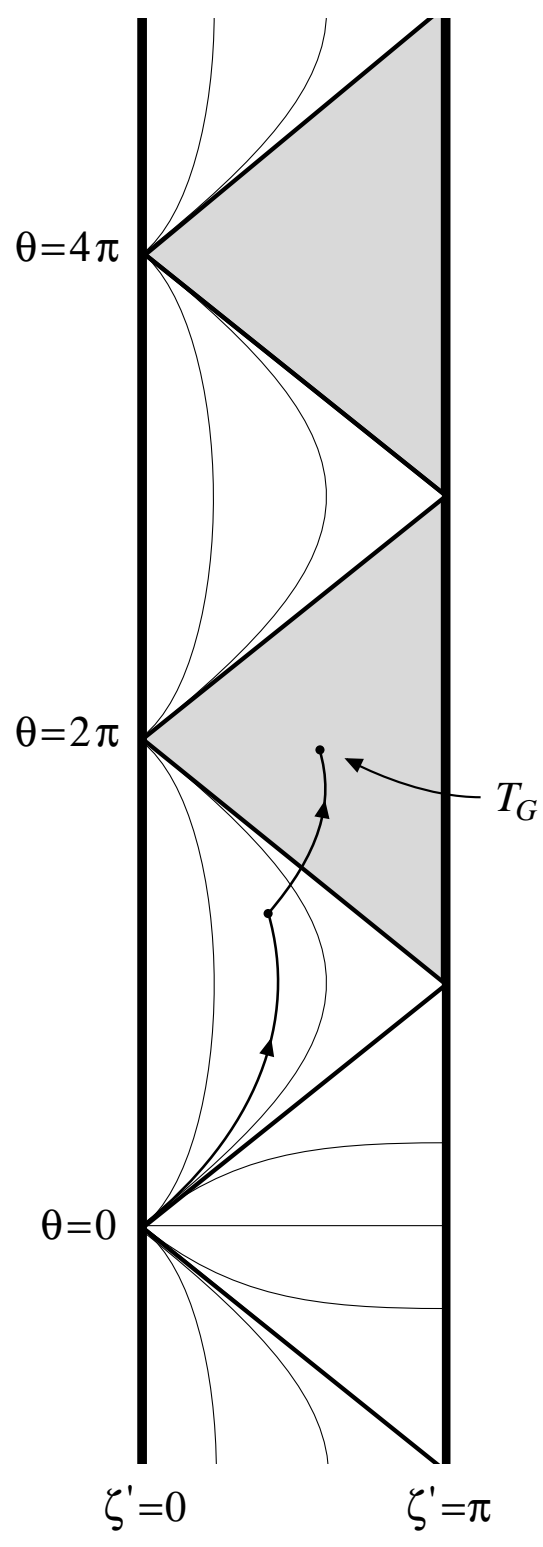

23 
specifying an element $g \in G$, and by specifying, up to continuous deformation, a path in $G$ connecting the identity element to $g$. To make use of this fact, imagine that the mass of each particle is rendered nonsingular by smearing it over a small region. To define the $\widetilde{\mathrm{SU}}(1,1)$ holonomy of a loop $C$, start with a trivial loop in $D_{C}$ (i.e., one which encircles no particles), and deform it through $D_{C}$ into the loop $C$. At each stage of the deformation there is an unambiguously defined holonomy element of $\mathrm{SO}(2,1)$, and therefore the deformation produces a continuous path in $\mathrm{SO}(2,1)$ from the identity to the holonomy group element for the loop $C$. Although the precise path in $\mathrm{SO}(2,1)$ will depend on the loop deformation that is chosen arbitrarily, the specification of $D_{C}$ guarantees that the path will be determined up to continuous deformation, precisely what is needed to define an element of the covering group $\widetilde{\mathrm{SU}}(1,1)$. Finally, this definition can be shown equivalent to the one given two paragraphs above by considering in particular the deformation of the loop which starts as a trivial loop, and expands to cover each of the particles one at a time, in the same order as the factors appearing in Eq. (3).

Since the element $T_{t o t} \in \widetilde{\mathrm{SU}}(1,1)$ depends only on $C$ and $D_{C}$, relationships derived by the continuous deformation of loops, such as the conservation law of Eq. (4) or the isolation of a subsystem described by Eq. (37), are valid equations in $\widetilde{S U}(1,1)$.

We can now describe the key step in our argument that a Gott time machine cannot be created from initial conditions specified on an open spacelike hypersurface with timelike total momentum. Consider an arbitrary subset (possibly the entire set) of particles crossing a spacelike hypersurface $S$, with a momentum characterized by a holonomy element $T_{\text {sub }}$ of $\widetilde{\mathrm{SU}}(1,1)$. Then (37) implies that the holonomy of all particles crossing $S$ can be written as

$$
T_{t o t}=T_{N} \ldots T_{1} T_{s u b}
$$

where the $T_{i}$ represent the individual particles comprising the rest of the system. The right hand side of this expression must correspond to a point in the future light cone of $T_{s u b}$, since each $T_{i}$ can be represented by a segment of a future-directed timelike or null geodesic. For an open hypersurface with timelike total momentum, however, $T_{t o t}$ in the rest frame corresponds to a rotation by the total deficit angle, which we will show must be less than or equal to $2 \pi$. Thus, $T_{t o t}$ must lie on the $\zeta^{\prime}=0$ axis, between $\theta=0$ and $\theta=2 \pi$. However, the holonomy element of a Gott time machine, $T_{G}$, lies in the shaded region that is spacelike separated from the point $\left(\theta=2 \pi, \zeta^{\prime}=0\right)$, as shown in Fig. 6 . Since $T_{\text {sub }}$ must lie to the past of $T_{t o t}$, $T_{\text {sub }}$ cannot represent the momentum of a Gott time machine. Thus, no subset of the particles crossing $S$ can comprise a Gott time machine.
To complete the argument, we must investigate the circumstances under which one can find a spacelike hypersurface $S$, as was used in the previous argument. The complication is the possibility of CTC's, which cannot be discounted a priori, as we are trying to prove that they cannot be created. In the presence of CTC's a spacetime will not necessarily admit a foliation into spacelike hypersurfaces, so it may not be possible to choose a holonomy loop so that all of the particles contribute positively. In addition, we must prove that the total deficit angle of an open hypersurface must be less than or equal to $2 \pi$. These steps are completed in the next section.

\section{RESTRICTIONS ON TIME MACHINE CONSTRUCTION}

In this section we consider an attempt to build a Gott time machine in a universe that is open, in the sense that it contains ${ }^{9}$ an edgeless spacelike surface $S$ with the topology of $\mathbb{R}^{2}$. We will further limit our attention to the case where $S$ is acausal (no timelike or null curve intersects $S$ more than once); in that case $S$ is called a partial Cauchy surface. Then we can speak of the total momentum of the particles passing through $S$, defined by the element $T_{S}$ of $\widetilde{\mathrm{SU}}(1,1)$ computed from the holonomy of a non-self-intersecting loop in $S$ at infinity, enclosing all of the particles. When all of the particles in the universe intersect $S$, we may think of $T_{S}$ as defining the total momentum of the universe; we will usually consider this situation, although it is not strictly necessary to our argument. In the case where $T_{S}$ is timelike, we will show that a group of particles with the momentum of a Gott time machine can evolve from the data specified on $S$ only if the rest frame deficit angle is greater than $2 \pi$. We then show that this deficit angle must be less than or equal to $2 \pi$ if $S$ has the topology of $\mathbb{R}^{2}$, i.e., if the $(2+1)$-dimensional universe is open.

We begin with some basic definitions; more complete discussions can be found in Refs. [2], [3], [5], and [21]. Consider a spacetime containing a partial Cauchy surface $S$. The future domain of dependence $D^{+}(S)$ is the set of all points $p$ such that every past-directed inextendible timelike or null curve through $p$ intersects $S$. Thus, initial data specified on $S$ suffice to determine the evolution throughout

9 In the absence of CTC's, it is reasonable to define open universes as those which may be foliated by spacelike surfaces with $\mathbb{R}^{2}$ topology. Such a foliation may not be possible when CTC's are present, however, even in spacetimes which we would intuitively classify as "open." Hence, we will take the existence of a single such surface as the defining characteristic of an open universe. 
$D^{+}(S)$. The past domain of dependence, and analogous terminology, is defined in the obvious way and denoted by replacing the plus sign by a minus sign. The full domain of dependence, $D(S)$, is defined as $D^{+}(S) \cup D^{-}(S)$. The future boundary of $D^{+}(S)$, past which information specified on $S$ is no longer sufficient to determine the evolution, is a null surface called the future Cauchy horizon $H^{+}(S)$. There are various circumstances under which a Cauchy horizon may arise, including the creation of CTC's. Any point $p$ that lies on a CTC in the future of $S$, or in the future of any such CTC, is not contained in the domain of dependence $D^{+}(S)$, since there will exist a past-directed inextendible timelike curve through $p$ which wraps forever around a CTC without ever intersecting $S$.

We also define a partial ordering in the group $\widetilde{\mathrm{SU}}(1,1)$ : we say that $T_{i}<T_{j}$ if and only if $T_{i}$ lies in the past light cone of $T_{j}$, in the anti-de Sitter metric of Eq. (21). The light cones used in this definition are easily visualized by using the conformal diagram of Fig. 6.

We will show that if the momentum associated with $S$ is timelike, then no Gott time machine can evolve from the particles passing through $S$. That is, no group of particles in the future domain of dependence $D^{+}(S)$, or on the Cauchy horizon $H^{+}(S)$, can have the holonomy of a Gott time machine. Thus, either the Cauchy horizon does not exist, or it arises due to an effect other than the creation of a Gott time machine.

First let $S^{\prime}$ be any Cauchy surface for $D(S)$, and let $C$ be a non-self-intersecting loop on $S^{\prime}$, which defines a holonomy $T_{C}$. We may decompose $T_{S^{\prime}}$ into $T_{C}$ times the positive contributions of other particles passing through $S^{\prime}$, as in (37). Hence $T_{C}$ must be less than or equal to $T_{S^{\prime}}$, in the ordering defined above. But since $S^{\prime}$ is a Cauchy surface, the particles that cross $S^{\prime}$ are exactly those that cross $S$. The loop defining $T_{S^{\prime}}$ can therefore be deformed into the loop defining $T_{S}$ without crossing any particle worldlines. This deformation can be carried out even if the particles undergo merges or decays, so in all cases $T_{S^{\prime}}=T_{S}$. Thus, $T_{C} \leq T_{S}$.

Now consider the general case where $S^{\prime}$ is any connected edgeless spacelike surface in $D(S)$ and $C$ is again a non-self-intersecting loop on $S^{\prime}$. We will show that $T_{C} \leq T_{S}$ by showing that $T_{C}$ can be embedded in a Cauchy surface $S^{\prime \prime}$, reducing this situation to the case of the previous paragraph.

We begin by using the method of Geroch [22] to define ${ }^{10}$ a time coordinate $\lambda$

10 In Ref. [22], Geroch restricts his construction to the interior of $D(S)$. However, with the present definition of $D^{+}(S)$ (which follows Refs. [3] and [21] and differs slightly from that of Geroch) the condition that $S$ be edgeless implies that $D(S)$ on $D(S)$. Choose a measure on $D(S)$ so that the total volume of $D(S)$ is 1 , and let $V^{+}(p)$ denote the volume of the future of a point $p$ in this measure. Similarly, let $V^{-}(p)$ denote the volume of the past, and let $\lambda \equiv V^{-} / V^{+}$. Clearly $\lambda$ increases on any timelike curve. Geroch shows that $\lambda$ is continuous and that it takes all values from $-\infty$ to $\infty$ on any inextendible timelike curve.

We now construct a coordinate system on $D(S)$ as follows: let $(x, y)$ be coordinates for $S$, and then let $\left(\lambda_{0}, x, y\right)$ denote the point where $\lambda=\lambda_{0}$ on the integral curve of $\nabla \lambda$ through $(x, y)$. Since $S$ has the topology of $\mathbb{R}^{2}, D(S)$ has the topology of $\mathbb{R}^{3}$, and in particular is simply connected.

We now show that since $S^{\prime}$ is spacelike, edgeless, and connected, it divides $D(S)$ into two regions: the past and the future of $S^{\prime}$. For any point $p \in D(S)$, we let $\Gamma \subset D(S)$ be a curve from $p$ that intersects $S^{\prime}$ at least once, and we let $p^{\prime}$ denote the first such intersection point. The point $p$ will be said to lie to the future or the past of $S^{\prime}$ according to whether $\Gamma$ approaches $p^{\prime}$ from the future or the past side of $S^{\prime}$. This is well-defined, because if two curves $\Gamma$ and $\Gamma^{\prime}$ from $p$ approach $S^{\prime}$ from different sides, we would be able to join $\Gamma$ and $\Gamma^{\prime}$ by a curve through $S^{\prime}$ to obtain a closed curve. An arbitrarily small distortion would then produce a curve that crosses $S^{\prime}$ exactly once. But such a curve is impossible: the topology of $D(S)$ implies that the curve would be contractible, but the edgelessness of $S^{\prime}$ implies that the number of crossings can only change by an even number. ${ }^{11}$ One can similarly show that $S^{\prime}$ is achronal, since any nonspacelike curve connecting two points on $S^{\prime}$ could also be used to construct a closed curve that crosses $S^{\prime}$ exactly once.

The loop $C$ and the interior of $C$ in $S^{\prime}$ together comprise a compact region $D_{C}$. Within $D_{C}, \lambda$ assumes a maximum value $\lambda_{\max }$ and a minimum value $\lambda_{\min }$. The desired surface $S^{\prime \prime}$ can then be defined by the relation $\lambda=f(x, y)$, where the function $f(x, y)$ is defined by the following procedure, illustrated in Fig. 7. If for the given $x$ and $y$ there is a point $(\lambda, x, y) \in S^{\prime}$ with $\lambda_{\min } \leq \lambda \leq \lambda_{\max }$, then let $f(x, y)=\lambda$. If not, then the points $\left(\lambda_{\min }, x, y\right)$ and $\left(\lambda_{\max }, x, y\right)$ are both on the same side of $S^{\prime}$. If they are in the past, let $f(x, y)=\lambda_{\text {max }}$; if they are in the future let $f(x, y)=\lambda_{\min }$. Note that $f(x, y)$ is continuous, and is bounded by $\lambda_{\min } \leq f(x, y) \leq \lambda_{\max }$.

To complete the argument, we must show that $S^{\prime \prime}$ is a Cauchy surface for $D(S)$, i.e., that any inextendible timelike curve $\gamma$ intersects $S^{\prime \prime}$ exactly once. For any point $(\lambda, x, y)$, let $\tilde{\lambda}=\lambda-f(x, y)$. The surface $S^{\prime \prime}$ is then described by $\tilde{\lambda}=0$,

is open. The construction, therefore, applies to all of $D(S)$.

11 See, for example, p. 204 of Ref. [3]. 


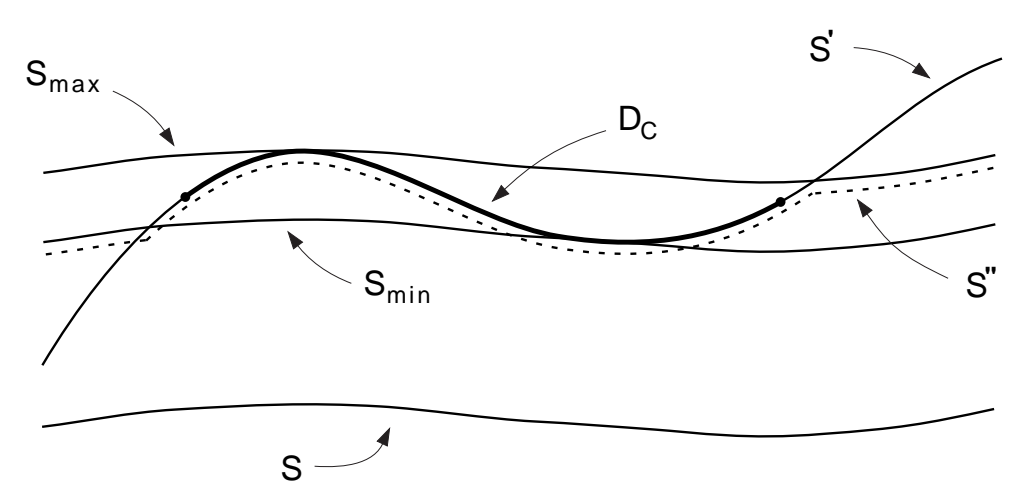

Fig. 7: The construction of the Cauchy surface $S^{\prime \prime}$. Given an edgeless spacelike surface $S^{\prime}$ in $D(S)$ and a closed region $D_{C} \subset S^{\prime}$, the procedure described in the text serves to define another surface $S^{\prime \prime}$ such that $D_{C} \subset$ $S^{\prime \prime}$, and $S^{\prime \prime}$ is a Cauchy surface for $D(S)$. In this figure, $S_{\min }$ and $S_{\max }$ are surfaces defined by $\lambda=\lambda_{\min }$ and $\lambda=\lambda_{\max }$, respectively, where $\lambda_{\min }$ and $\lambda_{\max }$ are the minimum and maximum values of the function $\lambda$ on the region $D_{C}$.

with $\tilde{\lambda}$ positive in the future and negative in the past. Since $\lambda$ takes all values from $-\infty$ to $\infty$ along the curve $\gamma$, the same must be true for $\tilde{\lambda}$, and hence $S^{\prime \prime}$ must be intersected at least once. Following $\gamma$ toward the future, we see that at the first crossing of $S^{\prime \prime}, \tilde{\lambda}$ changes from a negative value to a positive one. But then there can be no further crossings, since at the next crossing $\tilde{\lambda}$ would have to change from positive to negative, which implies that $\gamma$ would cross the spacelike surface $S^{\prime \prime}$ toward the past. Thus there is exactly one intersection of $\gamma$ and $S^{\prime \prime}$, so $S^{\prime \prime}$ is a Cauchy surface for $D(S)$. Since $C$ lies on $S^{\prime \prime}$, we find by the earlier argument that $T_{C} \leq T_{S}^{\prime \prime}=T_{S}$.

An equivalent result holds for particles passing through the Cauchy horizon. ${ }^{12}$ To see this, consider a non-self-intersecting loop $C$ on $H^{+}(S)$. We will deform $C$ into the interior of $D(S)$, where the previous result can be used. Let $V$ be any continuous timelike vector field defined on $D^{+}(S) \cup H^{+}(S)$. From each point of $C$, follow the integral curve of $V$ backward through $D^{+}(S)$ until it reaches a surface

$12 H^{+}(S)$ is null, rather than spacelike, but the definition of the holonomy of a system of particles can be trivially extended to this case, as long as no particle worldline lies on $\mathrm{H}^{+}(S)$. If such a particle worldline did exist, the holonomy would not be defined, since the holonomy loop would intersect it.

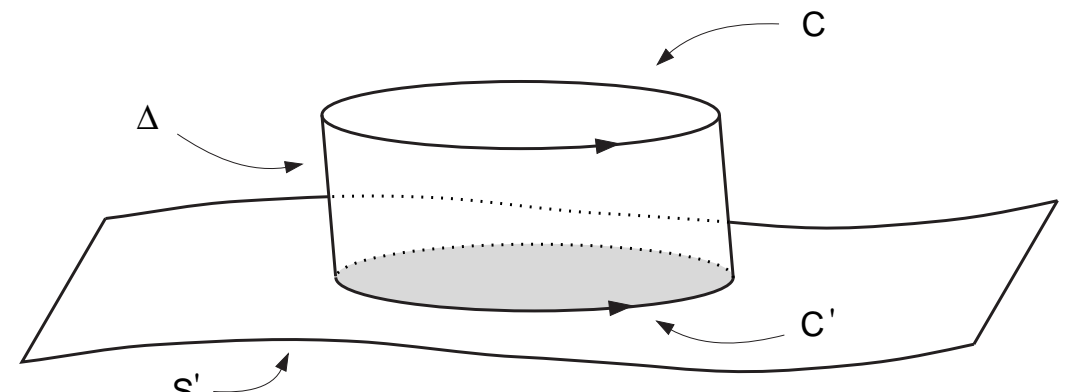

$S^{\prime}$

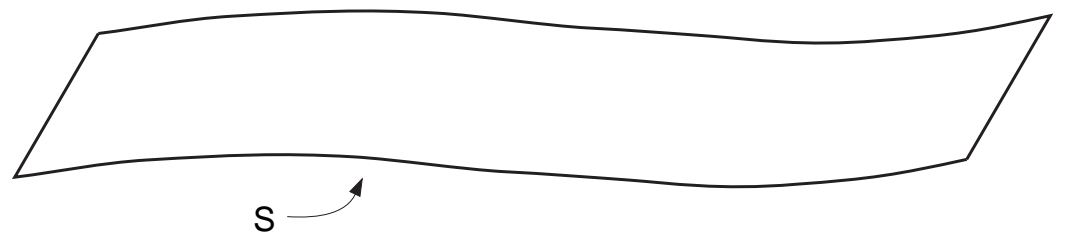

Fig. 8: Holonomy of a loop on the Cauchy horizon. The loop $C$ is joined by a timelike cylinder $\Delta$ to a loop $C^{\prime}$ embedded in $S^{\prime}$, a spacelike surface in the domain of dependence of $S$. No particles pass through $\Delta$, so the holonomy of $C$ is equal to that of $C^{\prime}$, which arises from the contributions of particles passing through the shaded region, the interior of $C^{\prime}$ in $S^{\prime}$.

$S^{\prime}$ defined by $\lambda=\lambda^{\prime}$, where $\lambda^{\prime}$ is a constant. Call the resulting loop $C^{\prime}$, and let $\Delta$ be the cylinder that connects $C$ to $C^{\prime}$, as shown in Fig. 8. Choose $\lambda^{\prime}$ large enough (i.e., $S^{\prime}$ late enough) so that no particle worldline intersects $\Delta$. The holonomy $T_{C}$ is then equal to $T_{C^{\prime}}$, which is less than or equal to $T_{S}$ by the argument above.

We will now show that any spacetime containing a complete spacelike surface with the topology of $\mathbb{R}^{2}$ and a timelike total momentum must have a rest frame deficit angle less than or equal to $2 \pi$. We first consider the case of an arbitrary 2-dimensional surface that is flat everywhere except for the interior of a loop $L$; only the intrinsic properties of the surface will concern us in this paragraph. Let $\Sigma$ be the interior of $L$, and $K$ be the scalar curvature of $\Sigma$. The Gauss-Bonnet theorem (see, e.g., [23]) tells us that, for surfaces with the topology of $\mathbb{R}^{2}$,

$$
\int_{\Sigma} K d A=2 \pi-\int_{L} \frac{d \phi}{d s} d s
$$

Here, $\phi$ is the angle between the tangent vector of $L$ and an arbitrary vector parallel transported along $L$ in the 2-dimensional surface. We call the right hand side of this 
equation the turning deficit angle $\theta_{\text {turn }}$, and we will show below that it is equal to the holonomy deficit angle $\theta$, defined earlier. As the surface is flat outside the loop (by hypothesis), it follows that the left hand side of Eq. (39) gives the integrated curvature over the entire surface. We can then invoke a theorem of Cohn-Vossen [13], which states that the integrated curvature of a geodesically complete surface with the topology of $\mathbb{R}^{2}$ is less than or equal to $2 \pi$, from which it follows that

$$
\theta_{\text {turn }} \equiv 2 \pi-\int_{L} \frac{d \phi}{d s} d s \leq 2 \pi
$$

Thus, a two-dimensional surface that is flat outside of a loop $L$ and has $\mathbb{R}^{2}$ topology necessarily has a turning deficit angle less than or equal to $2 \pi$.

Our task, then, is to find such a surface in our spacetime. The surface $S$ whose existence we have hypothesized is not necessarily sufficient - even though the portion of $S$ that lies outside the loop $L$ is locally embedded in Minkowski space, there is no guarantee that it is intrinsically flat, and it is therefore hard to apply the above reasoning. However, it is possible to define a flat metric on this portion of $S$, by the following procedure. Let $\hat{t}$ denote the future-directed unit vector left invariant by the holonomy around $L$. If the holonomy is a rotation by a multiple of $2 \pi$, then choose $\hat{t}$ to be any future-directed timelike unit vector. In either case, $\hat{t}$ can be consistently parallel transported throughout the portion of $S$ exterior to $L$. In a (three-dimensional) neighborhood of any point on $S$, we can construct a Minkowskian coordinate system $(t, x, y)$ with metric $d s^{2}=-d t^{2}+d x^{2}+d y^{2}$, such that the direction of the $t$-axis coincides with $\hat{t}$. Since $S$ is spacelike, we may use $(x, y)$ as coordinates on $S$ locally, and then define the metric on the region covered by these coordinates to be $d x^{2}+d y^{2}$. Note that this need not agree with the induced metric from the spacetime, as $S$ may be curved in the latter metric. Note also that the flat metric is uniquely defined: the local Minkowskian coordinate system is specified up to translations or rotations in the $x-y$ plane, under which the metric $d x^{2}+d y^{2}$ is invariant. We thus have a well-defined flat metric on $S$, outside the loop $L$.

Now we must show that the turning deficit angle $\theta_{\text {turn }}$ is equal to the holonomy deficit angle $\theta_{S}$, defined by the rotation angle of $T_{S} \in \widehat{S U}(1,1)$ in its rest frame. For this purpose we will use the alternative definition discussed in Sec. II for defining the holonomy $T_{S}$ in the universal covering group. By this definition, the ambiguity of rotations by $2 \pi$ that occurs in $\mathrm{SO}(2,1)$ is resolved by continuous deformation of the loop. We introduce a 1-parameter class of loops $L_{\lambda}$, each with the same base point $Q$, where $L_{0}$ is a trivial loop that encircles no particles and $L_{1}=L$. As in Sec. II we imagine that the mass of each particle is smeared over a small region, so the holonomy changes continuously as the loop is varied. Let $T_{\lambda}$ denote the holonomy of $L_{\lambda}$, and note that it is uniquely defined in $\widetilde{\mathrm{SU}}(1,1)$ by requiring that it be continuous in $\lambda$, with $T_{0} \equiv I$ (the identity element). For definiteness, we describe the loop $L_{\lambda}$ in parameterized form as $L_{\lambda}(s)$, where $0 \leq s \leq 1$. Let $V_{\lambda}(s)$ denote the tangent vector of $L_{\lambda}$ at $s$, mapped to the tangent space at $Q$ by parallel transporting backward (i.e., clockwise) along $L_{\lambda}$. Thus, $V_{\lambda}(s)$ traces out a continuous curve in the tangent space at $Q$. The tangent vector must return to its original value at $s=1$, but $V_{\lambda}(1)$ is defined so that its value is modified by parallel transport clockwise around the loop $L_{\lambda}$. Inverting this transformation, we have

$$
T_{\lambda} V_{\lambda}(1)=V_{\lambda}(0) \text {. }
$$

Eq. (41) allows us to define a continuous loop in the tangent space at $Q$ by noting that $\widetilde{S U}(1,1)$ is simply connected, so a curve $g_{\lambda}(s)$ in $\widetilde{S U}(1,1)$ satisfying $g_{\lambda}(1)=I$ and $g_{\lambda}(2)=T_{\lambda}$ can be constructed uniquely, up to continuous deformation. Then define a closed curve $\bar{V}_{\lambda}(s)$ in the tangent space at $Q$ by concatenating $V_{\lambda}(s)$ and $g_{\lambda}(s) V_{\lambda}(1)$ :

$$
\bar{V}_{\lambda}(s) \equiv \begin{cases}V_{\lambda}(s) & \text { if } 0 \leq s \leq 1 \\ g_{\lambda}(s) V_{\lambda}(1) & \text { if } 1 \leq s \leq 2 .\end{cases}
$$

$\bar{V}_{\lambda}(s)$ is confined to the spacelike part of the tangent space, which is not simply connected (note that the zero vector is not spacelike). $\bar{V}_{0}(s)$ makes one counterclockwise loop around the origin as $s$ is varied from 0 to 2 , so by continuous deformation the same statement must hold for hold for all $\lambda$, and in particular for $\lambda=1$.

Now we must connect the behavior of the 3D tangent vector $V$ (defined in the $(2+1)$-dimensional tangent space at $Q)$ to the 2D tangent vector in the surface $S$, which was used to define the turning angle. We use the same local Minkowskian coordinate system $(t, x, y)$ that was used to construct the flat metric, and again we take $x$ and $y$ to locally define coordinates on $S$. The components of the 2D tangent vector are then equal to the $x$ and $y$ components of the 3D tangent vector, and in both the 2D and 3D spaces these components are unchanged by parallel transport. For any infinitesimal segment of loop, the turning angle $d \phi$ of the $2 \mathrm{D}$ tangent vector, as calculated in the flat metric, is equal to the change in the azimuthal angle of the 3D tangent vector (i.e., the angular change of the projection of the 3D tangent vector into the $x-y$ plane). Thus, the integrated change in the azimuthal angle of the tangent vector $\bar{V}_{1}(s)$ for $0 \leq s \leq 1$ is equal to the total turning angle,

$$
\int_{L} \frac{d \phi}{d s} d s \text {. }
$$


Since $g_{1}(1)=I$ and $g_{1}(2)=T_{S}$, the integrated change in the azimuthal angle of $\bar{V}_{1}(s)$ for $1 \leq s \leq 2$ is equal to the holonomy deficit angle $\theta_{S}$. Since $\bar{V}_{\lambda}$ is known to make one counterclockwise loop around the origin, we have

$$
\int_{L} \frac{d \phi}{d s} d s+\theta_{S}=2 \pi
$$

which shows that $\theta_{\text {turn }}=\theta_{S}$.

In the case at hand, this suffices to show that the holonomy $T_{S}$ must lie on the conformal diagram of $\widetilde{S U}(1,1)$ in the triangle to the future of the identity and the past of the region describing Gott time machines. Since we have argued that the holonomy of any group of particles in the domain of dependence of $S$, or on the Cauchy horizon (if it exists), must have a holonomy less than or equal to $T_{S}$, it is not possible for these particles to comprise a Gott time machine. We have therefore established that Gott time machines cannot be created in open universes with timelike total momentum.

\section{CONCLUSIONS}

The role played by closed timelike curves is an important issue in classical general relativity, and may be important in an ultimate quantum version of the theory. The general theorems of Tipler and Hawking are strong statements about the difficulty of creating CTC's, but incomplete in that they do not specify what will go wrong with any particular attempt at time machine construction. In this paper we have studied some specific obstacles to the creation of time machines of the type discovered by Gott [8], using the considerable simplification afforded by working in the toy model of $(2+1)$-dimensional gravity. These obstacles are most easily understood by considering the anti-de Sitter geometry of the 3-dimensional Lorentz group, in which we find that Gott time machines cannot lie to the past of collections of particles with timelike momentum and deficit angle less than $2 \pi$. We then use this fact to show that a Gott time machine cannot be created in an open universe with a timelike total momentum, essentially because there can never be enough energy in an open universe to achieve the Gott condition.

This result is situated within an ongoing discourse concerning the appearance and significance of CTC's in general relativity, including discussions of whether physics can be consistent in the presence of CTC's [24]. Considerable effort has recently been invested in understanding the creation of CTC's in (3+1)-dimensional spacetimes with traversable wormholes [25]. Such spacetimes seem to easily develop
CTC's, but the maintenance of a traversable wormhole requires violation of the weak energy condition (WEC). While quantum field theory in curved spacetime can allow WEC violation, there is evidence that quantum fluctuations serve to destabilize the would-be time machine, preventing the appearance of CTC's $[4,26]$. This issue has led to several investigations of the behavior of quantum fields on background spacetimes with CTC's [27]. These studies, which ask whether CTC creation is possible when the WEC is relaxed, are complementary to the one presented in this paper, which examines the nature of obstacles to CTC creation when the WEC is enforced.

It is unclear, however, what the implications of our result are for CTC creation in the real $(3+1)$-dimensional world. We have seen that in $(2+1)$ dimensions, where the unique property that spacetime is flat in vacuum precludes the possibilities of black hole creation and energy loss through gravitational radiation, this same property leads to a restriction on the total energy of an open universe with timelike total momentum, which in turn presents insuperable obstacles to time machine creation. However, the notion of a timelike momentum is rather intimately connected with the nature of the $(2+1)$-dimensional theory, so it seems inevitable that the TiplerHawking theorems in $(3+1)$ dimensions must be enforced by other means. Thus, a general understanding of the status of time machine creation remains elusive.

The issue of closed timelike curves in $(2+1)$ dimensions, moreover, has not been completely resolved; while we have pointed out in Section III that the TiplerHawking theorems apply in this context, there remains the possibility of time machines (distinct from the type proposed by Gott) with non-compactly generated Cauchy horizons. Waelbroeck [28] has shown that a two-particle system with timelike momentum does not support CTC's, and Kabat [29] has presented arguments suggesting that this result is more general. Menotti and Seminara [30] have discovered restrictions on the existence of time machines in stationary and axially symmetric spacetimes. A comprehensive proof (or counterexample) is worth searching for.

Meanwhile, the interpretation of holonomies in terms of the anti-de Sitter geometry of $\mathrm{SO}(2,1)$ sheds light on the "tachyonic" nature of the Gott two-particle system. While the energy-momentum vector of such a pair is properly described as spacelike, this fact does not render such a pair unphysical, as the energy-momentum vector does not tell the entire story. In the universal covering group of $\mathrm{SO}(2,1)$, the region containing Gott pairs is disjoint from that containing tachyons. The obstacle to creating a Gott time machine is not the tachyonic momentum as such, but the absence of sufficient energy in an open universe. This is seen most clearly by considering the case of closed universes. In our earlier paper [14] we argued 
that it is impossible to produce two particles satisfying the Gott condition by the decay of slowly-moving parent particles unless the total rest frame deficit angle exceeds $2 \pi$. In a closed universe, where the total deficit angle is $4 \pi$, this does not constitute an obstacle. It is easy to construct a closed universe containing two particles, each with deficit angle between $\pi$ and $2 \pi$, and a number of less massive spectator particles which bring the total deficit angle to $4 \pi$. Using the description of decays given in Ref. [14], we have found that the two massive particles can decay in such a way that each emits an offspring at sufficiently high velocity that the total momentum of the two fast-moving particles is tachyonic. ${ }^{13}$ Thus, in a closed (2+1)-dimensional universe it is possible to "build a tachyon." However, as we mentioned in the introduction, 't Hooft [17] has shown that the size of the universe begins to shrink after the decays, leading to a crunch (zero volume) before any CTC's can arise. There is thus a sense in which general relativity is flexible enough to permit tachyons, but works very hard to prevent time travel.

\section{ACKNOWLEDGEMENTS}

We thank Bruce Allen, Malcolm Anderson, Raoul Bott, Robert Finn, John Friedmann, Alex Lyons, Robert MacPherson, Don Page, Ted Pyne, Jonathan Simon, Isadore Singer, Gerard 't Hooft, and David Vogan for very helpful conversations. The work of S.M.C. was supported in part by funds provided by the U.S. National Aeronautics and Space Administration (NASA) under contracts NAGW-931 and NGT-50850 and the National Science Foundation under grant PHY/9206867; the work of S.M.C., E.F. and A.H.G. was supported in part by the U.S. Department of Energy (D.O.E.) under contract \#DE-AC02-76ER03069; and the work of K.D.O. was supported in part by the Texas National Research Laboratory Commission under grant \#RGFY93-278C.

\section{APPENDIX A: THE TIPLER/HAWKING THEOREMS}

In this Appendix we review the theorems of Tipler [5] and Hawking [4], who make rigorous the notion of "building a time machine in a local region of spacetime," and then show that such construction is impossible using only normal matter, in the absence of singularities. We also explain how the theorems may be extended to $(2+1)$ dimensions.

\footnotetext{
13 Our construction is described by 't Hooft in Ref. [17].
}
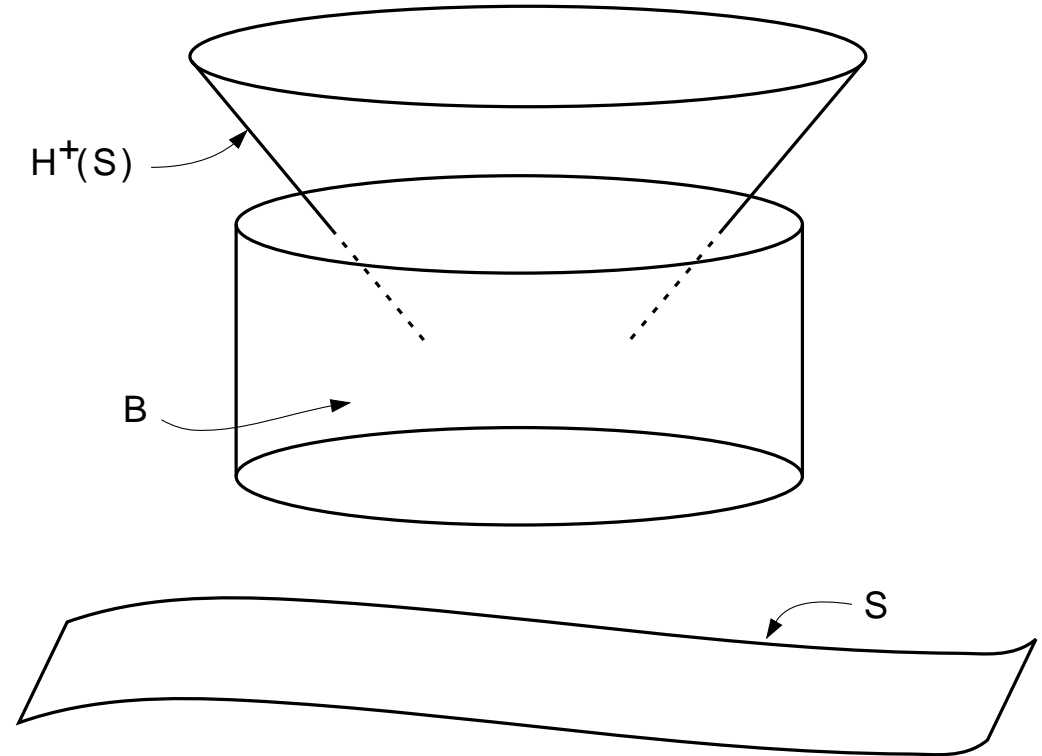

Fig. 9: Time machine creation in a local region. A partial Cauchy surface $S$ is pictured, in the future of which CTC's evolve. The Cauchy horizon, labelled $H^{+}(S)$, emerges from a compact set $B$.

The future Cauchy horizon $H^{+}(S)$, defined in Sec. III as the boundary of the future domain of dependence $D^{+}(S)$, is a null surface which can be thought of as the union of null geodesic segments known as "generators." The generators of $H^{+}(S)$ have no past endpoints, and they never intersect in the past. They may have future endpoints if the generators intersect; the set of such endpoints forms a set of measure zero. The notion of "creating a time machine in a local region" can be defined precisely by considering Cauchy horizons for which every generator, when followed into the past, enters a compact region of spacetime $B$ and remains there. (Note that a set which would otherwise be compact can be rendered non-compact by the appearance of a curvature singularity.) Hawking [4] refers to such horizons as "compactly generated." These are the types of time machines that could, in principle, be constructed by an advanced civilization. Since the generators can have no past endpoints, each generator entering the region $B$ must wind round and round within $B[4]$.

The situation is thus as we have portrayed in Fig. 9. A compact set $B$ lies in the future of a partial Cauchy surface $S$. The Cauchy horizon $H^{+}(S)$ emerges 
from a compact set $B$, which may be thought of as the place where CTC's are created. Tipler and Hawking essentially prove that this picture will never describe a spacetime obeying the weak energy condition (that the energy density measured by any timelike observer is nonnegative). Since we believe that "ordinary" matter obeys the weak energy condition, this theorem demonstrates that (in the absence of singularities) a time machine cannot be constructed in a local region - any attempt to do so will either fail, or render $B$ non-compact by creating a singularity (or both).

The theorems of Tipler [5] and Hawking [4] reach slightly different conclusions from slightly different assumptions. Tipler assumes that there are tidal forces somewhere on the Cauchy horizon inside $B$. Specifically, he requires at least one point $q$ on $H^{+}(S) \cap B$ at which $K^{\mu} K^{\nu} K_{[\sigma} R_{\rho] \mu \nu[\lambda} K_{\tau]} \neq 0$, where $K^{\mu}$ is the tangent vector to the generator of $H^{+}(S)$ at $q$, and square brackets denote antisymmetrization. (The connection between this condition and tidal forces is discussed in Ref. [3].) Hawking, on the other hand, assumes that the universe is open - i.e., that the surface $S$ is noncompact - without making any assumption about the existence of tidal forces. Thus, neither theorem applies to Taub-NUT space, which features a compactly generated Cauchy horizon without violating the weak energy condition, but which describes a closed universe free of tidal forces.

Both Tipler's and Hawking's theorems were originally formulated in the context of $(3+1)$-dimensional general relativity, but we may easily extend their analysis to the $(2+1)$-dimensional case. The only aspect of the proof that depends on the number of spacetime dimensions is the use of the (Newman-Penrose) optical scalar equations. These equations describe the behavior of a congruence of null geodesics in terms of scalar quantities, rather than the tensor quantities that appear in the Jacobi equation. In $(3+1)$ dimensions four scalars are required (the expansion, vorticity, and two components of shear), while in $(2+1)$ dimensions only one (the expansion) is needed. The Tipler-Hawking proof uses the equation obeyed by the expansion to show that the generators of a compactly generated Cauchy horizon must intersect in the past if the WEC is satisfied. However, it is a general property of future Cauchy horizons that the generators cannot intersect in the past, so the theorem is proven. The equation governing the behavior of the expansion in $(2+1)$ dimensions is obtained from the $(3+1)$-dimensional expression by omitting the shear and vorticity terms, as may easily be checked; it is then straightforward to show that the dimensionally reduced equation also implies that the generators of a compactly generated Cauchy horizon must intersect in the past. ${ }^{14}$ Therefore, the theorem

\footnotetext{
14 We are grateful to Ted Pyne for discussions on this point.
}

applies equally in $(2+1)$ or $(3+1)$ dimensions.

\section{APPENDIX B: SOME PROPERTIES OF SU(1,1)}

In this Appendix we demonstrate two technical properties concerning $\operatorname{SU}(1,1)$ and the embedding of its parameter space that is introduced in Sec. II-C. First, we prove the statement made in the text concerning the conditions under which a $2 \times 2$ matrix belongs to $\mathrm{SU}(1,1)$. Next we demonstrate the group invariance of the metric described in the text.

To derive the conditions under which a $2 \times 2$ matrix belongs to $\mathrm{SU}(1,1)$, we begin by parameterizing an arbitrary $2 \times 2$ matrix as

$$
T=\left(\begin{array}{ll}
a & b \\
c & d
\end{array}\right)
$$

where $a, b, c$, and $d$ are all complex. As stated in the text, $\operatorname{SU}(1,1)$ consists of those matrices $T$ satisfying

$$
\operatorname{det} T=+1
$$

and

$$
T^{\dagger} \eta T=\eta
$$

where

$$
\eta=\left(\begin{array}{cc}
1 & 0 \\
0 & -1
\end{array}\right) .
$$

From Eqs. (B.2) one has immediately that

$$
\begin{aligned}
& a^{*} a-c^{*} c=1 \\
& b^{*} b-d^{*} d=-1 \\
& b^{*} a-d^{*} c=0
\end{aligned}
$$

and

$$
a d-b c=1 .
$$

If Eq. (B.4c) is solved for $c$ and the result is inserted into (B.5), one finds

$$
a\left(d^{*} d-b^{*} b\right)=d^{*} .
$$


Using (B.4b), this reduces to

$$
a=d^{*} \text {. }
$$

Combining this result with Eq. (B.4c), one has

$$
b^{*}=c .
$$

Thus, $T$ can be written as

$$
T=\left(\begin{array}{cc}
a & b \\
b^{*} & a^{*}
\end{array}\right)
$$

where from Eq. (B.5) we have

$$
a^{*} a-b^{*} b=1 .
$$

Comparing with the parameterization

$$
T=\left(\begin{array}{ll}
w-i t & y+i x \\
y-i x & w+i t
\end{array}\right)
$$

used in the text, one sees that $w, t, x$, and $y$ are all real and satisfy

$$
-t^{2}+x^{2}+y^{2}-w^{2}=-1
$$

Conversely, it is easily shown that if $w, t, x$, and $y$ are all real and satisfy Eq. (B.11), then the matrix (B.10) belongs to $\mathrm{SU}(1,1)$.

Next, we wish to verify that the metric defined in Sec. II-C is group invariant. A group transformation on the group parameter space can be defined by mapping each element of the parameter space to the element obtained by multiplying on the left by a fixed element of the group, which we call $\tilde{T}$. Thus, the mapping is defined by

$$
\left(\begin{array}{cc}
w^{\prime}-i t^{\prime} & y^{\prime}+i x^{\prime} \\
y^{\prime}-i x^{\prime} & w^{\prime}+i t^{\prime}
\end{array}\right)=\tilde{T}\left(\begin{array}{ll}
w-i t & y+i x \\
y-i x & w+i t
\end{array}\right) .
$$

Note that the metric of Eq. (18) can be written as

$$
\begin{aligned}
d s^{2} & =-d t^{2}+d x^{2}+d y^{2}-d w^{2} \\
& =\operatorname{det}(d T)
\end{aligned}
$$

where

$$
d T=\left(\begin{array}{ll}
d w-i d t & d y+i d x \\
d y-i d x & d w+i d t
\end{array}\right)
$$

Since $\operatorname{det} \tilde{T}=1$, it follows immediately that $\operatorname{det}\left(d T^{\prime}\right)=\operatorname{det}(d T)$, so the metric is invariant. It is similarly clear that the metric is invariant under multiplication by a fixed group element on the right.

(It is not needed in our derivation, but it is interesting to note that the full invariance group of the metric given by $\mathrm{Eq} .(18)$ is $\mathrm{SO}(2,2)$, for which the Lie algebra is identical to $\mathrm{SU}(1,1) \times \operatorname{SU}(1,1)$. One of the two $\operatorname{SU}(1,1)$ subgroups has generators that are self-dual (in the 4-dimensional $w-t-x-y$ space), and the other has generators that are anti-self-dual. Transformations of the form described by Eq. (B.12) make up one of the $\mathrm{SU}(1,1)$ subgroups, while the other subgroup corresponds to multiplication on the right.)

\section{REFERENCES}

1. R. Geroch and G. T. Horowitz, in General Relativity: an Einstein Centenary Survey, eds. S. W. Hawking and W. Israel, (Cambridge Univ. Press, Cambridge, England, 1979).

2. K. S. Thorne, in General Relativity and Gravitation 1992, eds. R. J. Gleiser, C. N. Kozameh, and O. M. Moreschi, (Institute of Physics Publishing, Bristol, England, 1993).

3. S. W. Hawking and G. F. R. Ellis, The Large Scale Structure of Spacetime (Cambridge Univ. Press, Cambridge, England, 1973).

4. S. W. Hawking, Phys. Rev. D 46, 603 (1992).

5. F. J. Tipler, Phys. Rev. Lett. 37, 879 (1976); Ann. Phys. 108, 1 (1977). See particularly Theorem 3 of the latter paper.

6. S. L. Shapiro and S. A. Teukolsky, Phys. Rev. D 45, 2206 (1992).

7. A. Ori, Phys. Rev. Lett. 71, 2517 (1993).

8. J. R. Gott, Phys. Rev. Lett. 66, 1126 (1991).

9. For a review, see B. Allen and J. Simon, Nature 357, 19 (1992).

10. C. Cutler, Phys. Rev. D 45, 487 (1992); see also A. Ori, Phys. Rev. D 44, R2214 (1991).

11. L. Marder, Proc. R. Soc. A261, 91 (1961); A. Staruszkiewicz, Acta. Phys. Polon. 24, 734 (1963); J. R. Gott and M. Alpert, Gen. Rel. Grav. 16, 243 
(1984); S. Giddings, J. Abbott, and K. Kuchař, Gen. Rel. Grav. 16, 751 (1984).

12. S. Deser, R. Jackiw, and G. 't Hooft, Ann. Phys. 152, 220 (1984).

13. S. Cohn-Vossen, Compositio Math. 2, 69 (1935); see also A. Huber, Comment. Math. Helv. 32, 13 (1957).

14. S. M. Carroll, E. Farhi, and A. H. Guth, Phys. Rev. Lett. 68, 263 (1992); Erratum: 68, 3368 (1992).

15. E. Witten, Nucl. Phys. B311, 46 (1988); S. Carlip, Nucl. Phys. B324, 106 (1989); S. P. Martin, Nucl. Phys. B327, 178 (1989); D. Lancaster and N. Sasakura, Class. Quantum Grav. 8, 1481 (1991).

16. S. Deser, R. Jackiw, and G. 't Hooft, Phys. Rev. Lett. 68, 267 (1992).

17. G. 't Hooft, Class. Quantum Grav. 9, 1335 (1992); Class. Quantum Grav. 10, 1023 (1993).

18. S. Helgason, Differential Geometry and Symmetric Spaces (Academic Press, San Diego, 1962); J. A. Wolf, Spaces of Constant Curvature (McGraw-Hill, New York, 1967).

19. J. Balog, L. O'Raifeartaigh, P. Forgács, and A. Wipf, Nucl. Phys. B325, 225 (1989).

20. L. Pukánsky, Mathematische Annalen 156, 96 (1964).

21. R. Wald, General Relativity (University of Chicago Press, Chicago, 1984).

22. R.P. Geroch, J. Math. Phys. 11, 437 (1970); see also Ref. [3], especially proposition 6.6.8.

23. C. Nash and S. Sen, Topology and Geometry for Physicists (Academic Press, London, 1983).

24. V. P. Frolov and I. D. Novikov, Phys. Rev. D 42, 1057 (1990); J. Friedman, M. S. Morris, I. D. Novikov, F. Echeverria, G. Klinkhammer, K. S. Thorne, and U. Yurtsever, Phys. Rev. D 42, 1915 (1990); F. Echeverria, G. Klinkhammer, and K. S. Thorne, Phys. Rev. D 44, 1077 (1991); D. Deutsch, Phys. Rev. D 44, 3197 (1991); J. L. Friedman and M. S. Morris, Phys. Rev. Lett. 66, 401 (1991); J. L. Friedman, N. J. Papastamatiou, and J. Z. Simon, Phys. Rev. D 46, 4456 (1992); D. S. Goldwirth, M. J. Perry, T. Piran and K. Thorne, "The Quantum Propagator for a Nonrelativistic Particle in the Vicinity of a Time Machine," preprint gr-qc/9308009 (1993).
25. M. S. Morris, K. S. Thorne, and U. Yurtsever, Phys. Rev. Lett. 61, 1446 (1988).

26. S. W. Kim and K. S. Thorne, Phys. Rev. D 43, 3929 (1991).

27. D. G. Boulware, Phys. Rev. D 46, 4421 (1992); H. D. Politzer, Phys. Rev. D 46, 4470 (1992); M. Visser, Phys. Rev. D 47, 554 (1993); J. D. E. Grant, Phys Rev. D 47, 2388 (1993).

28. H. Waelbroeck, Gen. Rel. Grav. 23, 219 (1991); Nucl. Phys. B364, 475 (1991).

29. D. Kabat, Phys. Rev. D 46, 2720 (1992).

30. P. Menotti and D. Seminara, Phys. Lett. B301, 25 (1993); Erratum: B307, 404 (1993); P. Menotti and D. Seminara, Stationary Solutions and Closed Timelike Curves in 2+1 Dimensional Gravity, preprint hep-th/9305164. 